Published in final edited form as:

Nat Genet. 2012 September ; 44(9): 981-990. doi:10.1038/ng.2383.

\title{
Large-scale association analysis provides insights into the genetic architecture and pathophysiology of type 2 diabetes
}

\begin{abstract}
Andrew P Morris ${ }^{1,140}$, Benjamin F Voight ${ }^{2,3,140}$, Tanya M Teslovich ${ }^{4,140}$, Teresa Ferreira $^{1,140}$, Ayellet V Segré ${ }^{2,5,6,140}$, Valgerdur Steinthorsdottir ${ }^{7}$, Rona J Strawbridge ${ }^{8,9}$, Hassan Khan ${ }^{10}$, Harald Grallert ${ }^{11}$, Anubha Mahajan ${ }^{1}$, Inga Prokopenko ${ }^{1,12}$, Hyun Min Kang $^{4}$, Christian Dina ${ }^{13,15}$, Tonu Esko ${ }^{16,17}$, Ross M Fraser ${ }^{18}$, Stavroula Kanoni ${ }^{19}$, Ashish Kumar $^{1}$, Vasiliki Lagou', Claudia Langenberg ${ }^{20}$, Jian'an Luan ${ }^{20}$, Cecilia M Lindgren ${ }^{1}$, Martina Müller-Nurasyid ${ }^{21,23}$, Sonali Pechlivanis ${ }^{24}$, N William Rayner ${ }^{1,12}$, Laura J Scott ${ }^{4}$, Steven Wiltshire ${ }^{1}$, Loic Yengo 25,26 , Leena Kinnunen ${ }^{27}$, Elizabeth J Rossin ${ }^{2,5,28,29}$, Soumya Raychaudhuri ${ }^{2,30,31}$, Andrew D Johnson ${ }^{32}$, Antigone S Dimas ${ }^{1,33,34}$, Ruth J F Loos $^{20,35,36,37}$, Sailaja Vedantam ${ }^{38,39}$, Han Chen ${ }^{40}$, Jose C Florez ${ }^{5,6,38,41}$, Caroline Fox ${ }^{32,42}$, Ching-Ti Liu ${ }^{40}$, Denis Rybin ${ }^{43}$, David J Couper ${ }^{44}$, Wen Hong L Kao ${ }^{45}$, Man Li ${ }^{45}$, Marilyn C Cornelis $^{46}$, Peter Kraft ${ }^{46,47}$, Qi Sun ${ }^{46,48}$, Rob M van Dam ${ }^{46,49}$, Heather M Stringham ${ }^{4}$, Peter S Chines ${ }^{50}$, Krista Fischer ${ }^{16}$, Pierre Fontanillas ${ }^{2}$, Oddgeir L Holmen ${ }^{51}$, Sarah E Hunt ${ }^{19}$, Anne U Jackson ${ }^{4}$, Augustine Kong ${ }^{7}$, Robert Lawrence ${ }^{52}$, Julia Meyer ${ }^{22}$, John R B Perry ${ }^{1,53}$, Carl G P Platou ${ }^{51,54}$, Simon Potter ${ }^{19}$, Emil Rehnberg ${ }^{55}$, Neil Robertson ${ }^{1,12}$, Suthesh
\end{abstract}

\footnotetext{
Correspondence should be addressed to A.P.M. (amorris@well.ox.ac.uk), M.B. (boehnke@umich.edu) or M.I.M. (mark.mccarthy@drl.ox.ac.uk).

139 Deceased.

140 These authors contributed equally to this work.

${ }^{141}$ These authors jointly supervised the work.
}

AUTHOR CONTRIBUTIONS Writing group: A.P.M., B.F.V., T.M.T., T. Ferreira, A.V.S., V. Steinthorsdottir, R.J.S., H.K., H.G., A. Mahajan, I.P., M.B., M.I.M.

GWAS re-analysis: A.P.M., B.F.V., A.V.S., V. Steinthorsdottir, H.G., I.P., C.D., C.M.L., N.W.R., L.J.S., S.W., S. Raychaudhuri, H Chen, C.F., C. Liu, D.R., D.J.C., W.H.L.K., M. Li, C.M.C., P.K., Q.S., R.M.v.D., H.M.S., P.S.C., A. Kong, N.R., G.T., R.B., L.L.B., N.B., G.C., C.J.G., C. Guiducci, C.H., W.R., N.K., C. Sigurðsson, B.T., H. Campbell, C.v.D., A.G.U., A. Hofman, E.S., G.R.A., K.R.O., E.Z., B.B., C.N.A.P., V. Lyssenko, T. Tuomi, B.I., D.J.H., L.Q., M.R., J.F.W., F.S.C., K.L.M., R.N.B., J. Tuomilehto, S.C., P. Froguel, T.I., A.D.M., T.M.F., A.T.H., E.B., P.M.N., U.T., L.C.G., K. Stefansson, F.H., J.S.P., J. Dupuis, J.B.M., D.A., M.B., M.I.M. Metabochip design: B.F.V., H.M.K., G.R.A., D.A., M.B., M.I.M.

Metabochip samples: P.A., M.A., R.B., G.C., A.S.F.D., M.D., T. Forsen, B.G., C.H., A.B.H, A. James, A. Jonsson, W.R., J. Kravic, K.L., E.L., S. Männistö, B.M., L.R., J. Saramies, B.S., S. Shah, G. Sigurðsson G, A. Silveira, G. Steinbach, B.T., F.V., R.W., D.Z., M.D.T., N.G.F., J.G.E., B.B., C.N.A.P., V. Lyssenko, T.T., B.I., A.R.S., M.R., I.B., J.B., K. Hovingh, J.F.P., J.F.W., R.R., T.A.L., L.L., G.D., I.N., N.L.P., K. Khaw, N.J.W., S.M.K., T.E.S., T.W., E.K., J. Saltevo, M. Laakso, J. Kuusisto, A. Metspalu, F.S.C., K.L.M., R.N.B., J. Tuomilehto, B.O.B., C. Gieger, K. Hveem, S.C., P. Froguel, D.B., E. Tremoli, S.E. Humphries, D.S., J. Danesh, E.I., S. Ripatti, V. Salomaa, R.E., K.H.J., S. Moebus, A.P., T.I., U.dF., A. Hamsten, A.D.M., P.J.D., T.M.F., A.T.H., O.M., S. Kathiresan, P.M.N., P.D., U.T., L.C.G., K. Stefansson, D.A., M.B., M.I.M.

Metabochip genotyping: L.L.B., J.C., A.T.C., S.E., E.E., G.G.B, C.J.G., C. Guiducci, J.H., N.K., K. Krjutškov, C. Langford, S.L., G.M., T.W.M., M.P., J. Trakalo, W.W., A. Syvänen, L.P., M.M.N.

Metabochip analysis: A.P.M., B.F.V., T.M.T., T. Ferreira, A.V.S., V. Steinsthorsdottir, R.J.S., H.K., H.G., A. Mahajan, I.P., T.E., R.M.F., S. Kanoni, L.K., A. Kumar, V. Lagou, J.L., C.M.L., M.M., S. Pechlivanis, N.W.R., L.J.S., S.W., L.Y., H.M.S., P.S.C., K.F., P. Fontanillas, O.L.H., S.E. Hunt, A.U.J., A. Kong, R.L., J.M., J.R.B.P., C.G.P.P., S. Potter, E.R., N.R., S. Sivapalaratnam, S. Stančáková, K. Stirrups, G.T., E. Tikkanen, A.R.W., K.G.

Core and additional analyses: A.P.M., B.F.V., T.M.T., T. Ferreira, A.V.S., V. Steinsthorsdottir, R.J.S., H.K., H.G., A. Mahajan, I.P., E.J.R., S. Raychaudhuri, A.D.J., A.S.D., R.J.F.L., S.V., V.E., M.B., M.I.M.

Consortium management: A.P.M., B.F.V., T.M.T., H.G., C. Langenberg, J.C.F., H. Campbell, C.v.D., G.R.A., K.R.O., E.Z., C.N.A.P., V. Lyssenko, A.R.S., I.B., J.F.W., K.L.M., C. Gieger, S.C., P. Froguel, E.I., T.I., A.D.M., T.M.F., A.T.H., U.T., L.C.G., K. Stefansson, F.H., J.S.P., J.B.M., D.A., M.B., M.I.M.

COMPETING FINANCIAL INTERESTS Valgerdur Steinthorsdottir, Gudmar Thorleifsson, Unnur Thorsteinsdottir and Kari Stefansson are employees at deCODE genetics, a biotechnology company that provides genetic testing services, and own stock/stock options in the company. Jose Florez received consulting honoraria from Novartis, Lilly and Pfizer. Inês Barroso and spouse own stock in Glaxosmithkline and Incyte Ltd. 
Sivapalaratnam ${ }^{56}$, Alena Stančáková ${ }^{57}$, Kathleen Stirrups ${ }^{19}{ }^{\text {, Gudmar Thorleifsson }}{ }^{7}$, Emmi Tikkanen ${ }^{58,59}$, Andrew R Wood ${ }^{53}$, Peter Almgren ${ }^{60}$, Mustafa Atalay ${ }^{61}$, Rafn Benediktsson ${ }^{62,63}$, Lori L Bonnycastle ${ }^{50}$, Noël Burtt ${ }^{2}$, Jason Carey ${ }^{2}$, Guillaume Charpentier $^{64}$, Andrew T Crenshaw ${ }^{2}$, Alex S F Doney ${ }^{65,66}$, Mozhgan Dorkhan ${ }^{60}$, Sarah Edkins $^{19}$, Valur Emilsson 67 , Elodie Eury ${ }^{25}$, Tom Forsen ${ }^{68,69}$, Karl Gertow ${ }^{8,9}$, Bruna Gigante $^{70}$, George B Grant ${ }^{2}$, Christopher J Groves ${ }^{12}$, Candace Guiducci ${ }^{2}$, Christian Herder $^{71}$, Astradur B Hreidarsson ${ }^{63}$, Jennie Hui ${ }^{72,75}$, Alan James ${ }^{72,76,77}$, Anna Jonsson 60 , Wolfgang Rathmann ${ }^{78}$, Norman Klopp ${ }^{11}$, Jasmina Kravic ${ }^{60}$, Kaarel Krjutškov ${ }^{16}$, Cordelia Langford $^{19}$, Karin Leander ${ }^{70}$, Eero Lindholm ${ }^{60}$, Stéphane Lobbens ${ }^{25}$, Satu Männistö ${ }^{59}$, Ghazala Mirza ${ }^{1}$, Thomas W Mühleisen ${ }^{79,80}$, Bill Musk ${ }^{72,75,77,81}$, Melissa Parkin ${ }^{2}$, Loukianos Rallidis $^{82}$, Jouko Saramies ${ }^{83}$, Bengt Sennblad ${ }^{8,9}$, Sonia Shah ${ }^{84}$, Gunnar Sigurðsson ${ }^{63,67}$, Angela Silveira ${ }^{8,9}$, Gerald Steinbach ${ }^{85}$, Barbara Thorand ${ }^{86}$, Joseph Trakalo ${ }^{1}$, Fabrizio Veglia ${ }^{87}$, Roman Wennauer ${ }^{85}$, Wendy Winckler ${ }^{2}$, Delilah Zabaneh ${ }^{84}$, Harry Campbell ${ }^{18,88}$, Cornelia van Duijn ${ }^{89,90}$, Andre G Uitterlinden ${ }^{89,91}$, Albert Hofman ${ }^{89}$, Eric Sijbrands ${ }^{91}$, Goncalo R Abecasis ${ }^{4}$, Katharine R Owen ${ }^{12,92}$, Eleftheria Zeggini ${ }^{19}$, Mieke D Trip ${ }^{56}$, Nita G Forouhi $^{20}$, Ann-Christine Syvänen ${ }^{93}$, Johan G Eriksson ${ }^{59,68,94,95}$, Leena Peltonen2,19,58,59,139, Markus M Nöthen ${ }^{79,80}$, Beverley Balkau 96,97 , Colin N A Palmer 65,66 , Valeriya Lyssenko ${ }^{60}$, Tiinamaija Tuomi ${ }^{95,98}$, Bo Isomaa ${ }^{95,99}$, David J Hunter ${ }^{46,48}$, Lu Qi ${ }^{46,48}$, Wellcome Trust Case Control Consortium ${ }^{100}$, MAGIC Investigators ${ }^{100}$, GIANT Consortium $^{100}$, AGEN-T2D Consortium ${ }^{100}$, SAT2D Consortium ${ }^{100}$, Alan R Shuldiner ${ }^{101,103,}$ Michael Roden ${ }^{71,104}$, Ines Barroso ${ }^{19,105,106}$, Tom Wilsgaard ${ }^{107}$, John Beilby ${ }^{72,73,74}$, Kees Hovingh ${ }^{56}$, Jackie F Price ${ }^{18}$, James F Wilson ${ }^{18,88}$, Rainer Rauramaa ${ }^{108,109}$, Timo A Lakka $^{61,108}$, Lars Lind ${ }^{110}$, George Dedoussis ${ }^{111}$, Inger Njølstad ${ }^{107}$, Nancy L Pedersen ${ }^{55}$, Kay-Tee Khaw ${ }^{10}$, Nicholas J Wareham ${ }^{20}$, Sirkka M Keinanen-Kiukaanniemi ${ }^{112,113}$, Timo E Saaristo ${ }^{114,115}$, Eeva Korpi-Hyövälti ${ }^{116}$, Juha Saltevo ${ }^{117}$, Markku Laakso ${ }^{57}$, Johanna Kuusisto ${ }^{57}$, Andres Metspalu ${ }^{16,17}$, Francis S Collins ${ }^{50}$, Karen L Mohlke ${ }^{118}$, Richard N Bergman $^{119}$, Jaakko Tuomilehto $27,116,120,121$, Bernhard O Boehm ${ }^{122}$, Christian Gieger 22 , Kristian Hveem ${ }^{51}$, Stephane Cauchi ${ }^{25}$, Philippe Froguel ${ }^{25,123}$, Damiano Baldassarre ${ }^{87,124}$, Elena Tremoli 87,124 , Steve E Humphries ${ }^{125}$, Danish Saleheen ${ }^{10,126}$, John Danesh ${ }^{10}$, Erik Ingelsson ${ }^{55}$, Samuli Ripatti ${ }^{19,58,59}$, Veikko Salomaa ${ }^{59}$, Raimund Erbel ${ }^{127}$, Karl-Heinz Jöckel $^{24}$, Susanne Moebus ${ }^{24}$, Annette Peters ${ }^{86}$, Thomas Illig ${ }^{11,128}$, Ulf de Faire ${ }^{70}$, Anders Hamsten 8,9 , Andrew D Morris ${ }^{65,66}$, Peter J Donnelly ${ }^{1,129}$, Timothy M Frayling ${ }^{53}$, Andrew T Hattersley ${ }^{130}$, Eric Boerwinkle ${ }^{131,132}$, Olle Melander ${ }^{60}$, Sekar Kathiresan $2,5,133$, Peter M Nilsson $^{60}$, Panos Deloukas ${ }^{19}$, Unnur Thorsteinsdottir ${ }^{7,62}$, Leif C Groop ${ }^{60}$, Kari

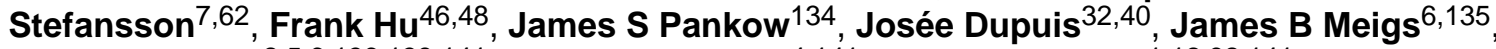
David Altshuler $2,5,6,136,138,141$, Michael Boehnke ${ }^{4,141}$, Mark I McCarthy ${ }^{1,12,92,141}$, and for the DIAGRAM Consortium

${ }^{1}$ Wellcome Trust Centre for Human Genetics, University of Oxford, Oxford, UK. ${ }^{2}$ Broad Institute of Harvard and Massachusetts Institute of Technology (MIT), Cambridge, Massachusetts, USA. ${ }^{3}$ University of Pennsylvania - Perelman School of Medicine, Department of Pharmacology, Philadelphia, Pennsylvania, USA. ${ }^{4}$ Department of Biostatistics, University of Michigan, Ann Arbor, Michigan, USA. ${ }^{5}$ Center for Human Genetic Research, Massachusetts General Hospital, Boston, Massachusetts, USA. ${ }^{6}$ Department of Medicine, Harvard Medical School, Boston, Massachusetts, USA. ${ }^{7}$ deCODE Genetics, Reykjavik, Iceland. ${ }^{8}$ Atherosclerosis Research Unit, Department of Medicine Solna, Karolinska Institutet, Stockholm, Sweden. ${ }^{9}$ Center for Molecular Medicine, Karolinska University Hospital Solna, Stockholm, Sweden. ${ }^{10}$ Department of Public Health and Primary Care, University of Cambridge, Cambridge, UK. ${ }^{11}$ Research Unit of Molecular Epidemiology, Helmholtz Zentrum Muenchen, Neuherberg, Germany. ${ }^{12}$ Oxford Centre for Diabetes, Endocrinology and Metabolism, University of Oxford, Oxford, UK. ${ }^{13}$ Inserm UMR 1087, Nantes, France. ${ }^{14}$ CNRS UMR 6291, Nantes, France. ${ }^{15}$ Nantes University, Nantes, France.

${ }^{16}$ Estonian Genome Center, University of Tartu, Tartu, Estonia. ${ }^{17}$ Institute of Molecular and Cell 
Biology, University of Tartu, Tartu, Estonia. ${ }^{18}$ Centre for Population Health Sciences, University of Edinburgh, Edinburgh, UK. ${ }^{19}$ Wellcome Trust Sanger Institute, Hinxton, Cambridge, UK. ${ }^{20} \mathrm{MRC}$ Epidemiology Unit, Institute of Metabolic Science, Addenbrooke's Hospital, Cambridge, UK. ${ }^{21}$ Institute of Medical Informatics, Biometry and Epidemiology, Ludwig-Maximilians-Universität, Munich, Germany. ${ }^{22}$ Institute of Genetic Epidemiology, Helmholtz Zentrum Muenchen, Neuherberg, Germany. ${ }^{23}$ Department of Medicine I, University Hospital Grosshadern, LudwigMaximilians-University, Munich, Germany. ${ }^{24}$ Institute for Medical Informatics, Biometry and Epidemiology, University Hospital of Essen, University Duisburg-Essen, Essen, Germany. ${ }^{25}$ CNRS-UMR-8199, Institute of Biology and Lille 2 University, Pasteur Institute, Lille, France. ${ }^{26}$ University Lille 1, Laboratory of Mathematics, CNRS-UMR 8524, MODAL team, INRIA Lille Nord-Europe, Lille, France. ${ }^{27}$ Diabetes Prevention Unit, National Institute for Health and Welfare, Helsinki, Finland. ${ }^{28} \mathrm{Health}$ Science and Technology MD Program, Harvard University and Massachusetts Institute of Technology, Boston, Massachusetts, USA. ${ }^{29} \mathrm{Harvard}$ Biological and Biomedical Sciences Program, Harvard University, Boston, Massachusetts, USA. ${ }^{30}$ Division of Rheumatology, Immunology and Allergy, Brigham and Women's Hospital, Harvard Medical School, Boston, Massachusetts, USA. ${ }^{31}$ Partners Center for Personalized Genomic Medicine, Boston, Massachusetts, USA. ${ }^{32}$ National Heart, Lung, and Blood Institute's Framingham Heart Study, Framingham, Massachusetts, USA. ${ }^{33}$ Department of Genetic Medicine and Development, University of Geneva Medical School, Geneva, Switzerland. ${ }^{34}$ Biomedical Sciences Research Center Al. Fleming, Vari, Greece. ${ }^{35}$ Charles R. Bronfman Institute for Personalized Medicine, Mount Sinai School of Medicine, New York, New York, USA. ${ }^{36}$ Child Health and Development Institute, Mount Sinai School of Medicine, New York, New York, USA. ${ }^{37}$ Department of Preventive Medicine, Mount Sinai School of Medicine, New York, New York, USA. ${ }^{38}$ Program in Medical and Population Genetics, Broad Institute, Cambridge, Massachusetts, USA. ${ }^{39}$ Division of Genetics and Endocrinology, Children's Hospital, Boston, Massachusetts, USA. ${ }^{40}$ Department of Biostatistics, Boston University School of Public Health, Boston, Massachusetts, USA. ${ }^{41}$ Diabetes Research Center, Diabetes Unit, Massachusetts General Hospital, Boston, Massachusetts, USA. ${ }^{42}$ Division of Endocrinology and Metabolism, Brigham and Women's Hospital and Harvard Medical School, Boston, Massachusetts, USA. ${ }^{43}$ Boston University Data Coordinating Center, Boston, Massachusetts, USA. ${ }^{44}$ Collaborative Studies Coordinating Center, Department of Biostatistics, University of North Carolina at Chapel Hill, Chapel Hill, North Carolina, USA. ${ }^{45}$ Department of Epidemiology, Johns Hopkins Bloomberg School of Public Health, Baltimore, Maryland, USA. ${ }^{46}$ Department of Nutrition and Epidemiology, Harvard School of Public Health, Boston, Massachusetts, USA. ${ }^{47}$ Program in Molecular and Genetic Epidemiology, Harvard School of Public Health, Boston, Massachusetts, USA. ${ }^{48}$ Channing Laboratory, Department of Medicine, Brigham and Women's Hospital and Harvard Medical School, Boston, Massachusetts, USA. ${ }^{49}$ Saw Swee Hock School of Public Health, National University of Singapore, Singapore. ${ }^{50}$ National Human Genome Research Institute, National Institutes of Health, Bethesda, Maryland, USA. ${ }^{51}$ HUNT Research Centre, Department of Public Health and General Practice, Norwegian University of Science and Technology, Levanger, Norway. ${ }^{52}$ Centre for Genetic Epidemiology and Biostatistics, The University of Western Australia, Nedlands, Australia. ${ }^{53}$ Genetics of Complex Traits, Institute of Biomedical and Clinical Science, Peninsula Medical School, University of Exeter, Magdalen Road, Exeter, UK. ${ }^{54}$ Department of Internal Medicine, Levanger Hospital, NordTrøndelag Health Trust, Levanger, Norway. ${ }^{55}$ Department of Medical Epidemiology and Biostatistics, Karolinska Institutet, Stockholm, Sweden. ${ }^{56}$ Department of Vascular Medicine, Academic Medical Center, University of Amsterdam, Amsterdam, The Netherlands. ${ }^{57}$ Department of Medicine, University of Eastern Finland and Kuopio University Hospital, Kuopio, Finland. ${ }^{58}$ Institute for Molecular Medicine Finland (FIMM), Helsinki, Finland. ${ }^{59}$ Department of Chronic Disease Prevention, National Institute for Health and Welfare, Helsinki, Finland. ${ }^{60}$ Lund University Diabetes Centre, Department of Clinical Science Malmö, Scania University Hospital, Lund University, Malmö, Sweden. ${ }^{61}$ Institute of Biomedicine, Physiology, University of Eastern Finland, 
Kuopio Campus, Kuopio, Finland. ${ }^{62}$ Faculty of Medicine, University of Iceland, Reykjavík, Iceland. ${ }^{63}$ Landspitali University Hospital, Reykjavík, Iceland. ${ }^{64}$ Endocrinology-Diabetology Unit, CorbeilEssonnes Hospital, Corbeil-Essonnes, France. ${ }^{65}$ Diabetes Research Centre, Biomedical Research Institute, University of Dundee, Ninewells Hospital, Dundee, UK. ${ }^{66}$ Pharmacogenomics Centre, Biomedical Research Institute, University of Dundee, Ninewells Hospital, Dundee, UK. ${ }^{67}$ Icelandic Heart Association, Kopavogur, Iceland. ${ }^{68}$ Department of General Practice and Primary Health Care, University of Helsinki, Helsinki, Finland. ${ }^{69}$ Vaasa Health Care Centre, Vaasa, Finland. ${ }^{70}$ Division of Cardiovascular Epidemiology, Institute of Environmental Medicine, Karolinska Institutet, Stockholm, Sweden. ${ }^{71}$ Institute for Clinical Diabetology, German Diabetes Center, Leibniz Center for Diabetes Research at Heinrich Heine University Düsseldorf, Düsseldorf, Germany. ${ }^{72}$ Busselton Population Medical Research Institute, Sir Charles Gairdner Hospital, Nedlands, Australia. ${ }^{73}$ PathWest Laboratory Medicine of Western Australia, QEII Medical Centre, Nedlands, Australia. ${ }^{74}$ School of Pathology and Laboratory Medicine, The University of Western Australia, Nedlands, Australia. ${ }^{75}$ School of Population Health, The University of Western Australia, Nedlands, Australia. ${ }^{76}$ Department of Pulmonary Physiology and Sleep Medicine, West Australian Sleep Disorders Research Institute, Queen Elizabeth II Medical Centre, Hospital Avenue, Nedlands, Australia. ${ }^{77}$ School of Medicine and Pharmacology, University of Western Australia, Nedlands, Australia. ${ }^{78}$ Institute of Biometrics and Epidemiology, German Diabetes Center, Leibniz Center for Diabetes Research at Heinrich Heine University Düsseldorf, Düsseldorf, Germany. ${ }^{79}$ Institute of Human Genetics, University of Bonn, Bonn, Germany. ${ }^{80}$ Department of Genomics, Life \& Brain Center, University of Bonn, Bonn, Germany. ${ }^{81}$ Respiratory Medicine, Sir Charles Gairdner Hospital, Nedlands, Australia. ${ }^{82}$ University General Hospital Attikon, Athens, Greece. ${ }^{83}$ South Karelia Central Hospital, Lappeenranta, Finland. ${ }^{84} \mathrm{UCL}$ Genetics Institute, Department of Genetics, Evolution and Environment, University College London, London, UK. ${ }^{85}$ Department of Clinical Chemistry and Central Laboratory, University of Ulm, Ulm, Germany. ${ }^{86}$ Institute of Epidemiology II, Helmholtz Zentrum Muenchen, Neuherberg, Germany. ${ }^{87}$ Centro Cardiologico Monzino, IRCCS, Milan, Italy. ${ }^{88} \mathrm{MRC}$ Institute of Genetics and Molecular Medicine at the University of Edinburgh, Western General Hospital, Edinburgh, UK. ${ }^{89}$ Department of Epidemiology, Erasmus University Medical Center, Rotterdam, The Netherlands. ${ }^{90}$ Netherland Genomics Initiative, Netherlands Consortium for Healthy Ageing and Centre for Medical Systems Biology, Rotterdam, The Netherlands. ${ }^{91}$ Department of Internal Medicine, Erasmus University Medical Center, Rotterdam, The Netherlands. ${ }^{92}$ Oxford National Institute for Health Research Biomedical Research Centre, Churchill Hospital, Oxford, UK. ${ }^{93}$ Molecular Medicine, Department of Medical Sciences, Uppsala University, Uppsala, Sweden. ${ }^{4}$ Unit of General Practice, Helsinki University General Hospital, Helsinki, Finland. ${ }^{95}$ Folkhälsan Research Center, Helsinki, Finland. ${ }^{96}$ INSERM CESP U1018, Villejuif, France. ${ }^{97}$ University Paris Sud 11, UMRS 1018, Villejuif, France. ${ }^{98}$ Department of Medicine, Helsinki University Hospital, University of Helsinki, Helsinki, Finland. ${ }^{99}$ Department of Social Services and Health Care, Jakobstad, Finland. ${ }^{100}$ The members of these consortia are listed in the Supplementary Note ${ }^{101}$ Division of Endocrinology, Diabetes and Nutrition, University of Maryland School of Medicine, Baltimore, Maryland, USA. ${ }^{102}$ Geriatric Research Education and Clinical Center, Baltimore Veterans Administration Medical Center, Baltimore, Maryland, USA. ${ }^{103}$ Program in Personalised and Genomic Medicine, University of Maryland School of Medicine, Baltimore, Maryland, USA. ${ }^{104}$ Department of Medicine/Metabolic Diseases, Heinrich Heine University Düsseldorf, Düsseldorf, Germany. ${ }^{105}$ University of Cambridge Metabolic Research Laboratories, Institute of Metabolic Science, Addenbrooke's Hospital, Cambridge, UK. ${ }^{106}$ NIHR Cambridge Biomedical Research Centre, Institute of Metabolic Science, Addenbrooke's Hospital, Cambridge, UK. ${ }^{107}$ Department of Community Medicine, Faculty of Health Sciences, University of Tromsø, Troms $\varnothing$, Norway. ${ }^{108}$ Kuopio Research Institute of Exercise Medicine, Kuopio, Finland. ${ }^{109}$ Department of Clinical Physiology and Nuclear Medicine, Kuopio University Hospital, Kuopio, Finland. ${ }^{110}$ Department of Medical Sciences, Uppsala University, Akademiska Sjukhuset, Uppsala, Sweden. ${ }^{111}$ Department 
of Dietetics-Nutrition, Harokopio University, Athens, Greece. ${ }^{112}$ Faculty of Medicine, Institute of Health Sciences, University of Oulu, Oulu, Finland. ${ }^{113}$ Unit of General Practice, Oulu University Hospital, Oulu, Finland. ${ }^{114}$ Finnish Diabetes Association, Tampere, Finland. ${ }^{115}$ Pirkanmaa Hospital District, Tampere, Finland. ${ }^{116}$ South Ostrobothnia Central Hospital, Seinäjoki, Finland. ${ }^{117}$ Department of Medicine, Central Finland Central Hospital, Jyväskylä, Finland. ${ }^{118}$ Department of Genetics, University of North Carolina, Chapel Hill, North Carolina, USA. ${ }^{119}$ Diabetes and Obesity Research Institute, Cedars-Sinai Medical Center, Los Angeles, California, USA. ${ }^{120}$ Red RECAVA Grupo RD06/0014/0015, Hospital Universitario La Paz, Madrid, Spain. ${ }^{121}$ Centre for Vascular Prevention, Danube-University Krems, Krems, Austria. ${ }^{122}$ Division of Endocrinology and Diabetes, Department of Internal Medicine, University Medical Centre Ulm, Ulm, Germany. ${ }^{123}$ Genomic Medicine, Imperial College London, Hammersmith Hospital, London, UK. ${ }^{124}$ Department of Pharmacological Sciences, University of Milan, Milan, Italy. ${ }^{125}$ Institute of Cardiovascular Science, University College London, London, UK. ${ }^{126}$ Center for NonCommunicable Diseases Pakistan, Karachi, Pakistan. ${ }^{127}$ Clinic of Cardiology, West German Heart Centre, University Hospital of Essen, University Duisburg-Essen, Essen, Germany. ${ }^{128}$ Hannover Unified Biobank, Hannover Medical School, Hannover, Germany. ${ }^{129}$ Department of Statistics, University of Oxford, Oxford, UK. ${ }^{130}$ Diabetes Genetics, Institute of Biomedical and Clinical Science, Peninsula Medical School, University of Exeter, Exeter, UK. ${ }^{131}$ Human Genetics Center, University of Texas Health Science Center at Houston, Houston, Texas, USA. ${ }^{132}$ Human Genome Sequencing Center at Baylor College of Medicine, Houston, Texas, USA.

${ }^{133}$ Cardiovascular Research Center, Massachusetts General Hospital, Boston, Massachusetts, USA. ${ }^{134}$ Division of Epidemiology and Community Health, University of Minnesota, Minneapolis, Minnesota, USA. ${ }^{135}$ General Medicine Division, Massachusetts General Hospital, Boston, Massachusetts, USA. ${ }^{136}$ Department of Genetics, Harvard Medical School, Boston, Massachusetts, USA. ${ }^{137}$ Department of Molecular Biology, Harvard Medical School, Boston, Massachusetts, USA. ${ }^{138}$ Diabetes Unit, Massachusetts General Hospital, Boston, Massachusetts, USA.

\section{Abstract}

To extend understanding of the genetic architecture and molecular basis of type 2 diabetes (T2D), we conducted a meta-analysis of genetic variants on the Metabochip involving 34,840 cases and 114,981 controls, overwhelmingly of European descent. We identified ten previously unreported T2D susceptibility loci, including two demonstrating sex-differentiated association. Genome-wide analyses of these data are consistent with a long tail of further common variant loci explaining much of the variation in susceptibility to T2D. Exploration of the enlarged set of susceptibility loci implicates several processes, including CREBBP-related transcription, adipocytokine signalling and cell cycle regulation, in diabetes pathogenesis.

Type 2 diabetes (T2D) is a chronic metabolic disease with multifactorial pathogenesis ${ }^{1}$. Although the genetic contribution to T2D is well recognized, the current set of 56 established susceptibility loci, identified primarily through large-scale genome-wide association studies (GWAS) ${ }^{2-11}$, captures at best $10 \%$ of familial aggregation of the disease. The characteristics (effect sizes and risk allele frequencies (RAF)) of the variants contributing to the "unexplained" genetic variance remain far from clear. At the same time, difficulties in inferring biological mechanisms from the variants of modest effect identified by GWAS have inhibited progress in defining the pathophysiological basis of disease susceptibility. One key question is whether characterization of increasing numbers of risk loci will provide evidence, at the functional level, that susceptibility involves a limited set of molecular processes. 
To extend the discovery and characterization of variants influencing T2D susceptibility, we performed large-scale genotyping using the Metabochip. This custom array of 196,725 variants was designed to facilitate cost-effective follow-up of nominal associations for T2D and other metabolic and cardiovascular traits, and to enhance fine-mapping of established loci ${ }^{12}$. The T2D-nominated component of Metabochip comprises 21,774 variants, including 5,057 "replication" SNPs that capture the strongest, independent (CEU $\left.r^{2}<0.2\right)$ autosomal association signals from the GWAS meta-analysis conducted by the DIAbetes Genetics Replication and Meta-analysis (DIAGRAM) Consortium. This genome-wide meta-analysis ("DIAGRAMv3") includes data from 12,171 cases and 56,862 controls of European descent imputed up to 2.5 million autosomal SNPs, and augments the previously published "DIAGRAMv2" meta-analysis ${ }^{4}$ with four additional GWAS (Supplementary Table 1). The T2D-nominated content of Metabochip includes a further 16,717 variants, most chosen from 1000 Genomes Project pilot data ${ }^{13}$, to fine-map 27 established susceptibility loci.

\section{RESULTS}

\section{Study overview}

Our primary investigation combined the DIAGRAMv3 ("Stage 1") GWAS meta-analysis with a "Stage 2" meta-analysis comprising 22,669 cases and 58,119 controls genotyped with Metabochip, including 1,178 cases and 2,472 controls of Pakistani descent (PROMIS) (Online Methods and Supplementary Table 1). There was little evidence of heterogeneity in allelic effects between European- and Pakistani-descent studies in Stage 2 (Supplementary Fig. 1), so we report the combined meta-analysis including PROMIS with genomic control correction.

\section{T2D susceptibility loci reaching genome-wide significance}

Combining Stage 1 and Stage 2 meta-analyses (Supplementary Fig. 2), we identified eight new T2D susceptibility loci at genome-wide significance $\left(P<5 \times 10^{-8}\right)$ (Table 1 , Supplementary Fig. 3 and Supplementary Table 2). By convention, we have labelled loci according to the gene nearest to the lead SNP, unless a compelling biological candidate maps nearby. The strongest signals mapped to $Z M I Z 1\left(P=1.0 \times 10^{-10}\right), A N K 1(P=2.5 \times$ $\left.10^{-10}\right)$, and the region flanking $K L H D C 5\left(P=6.1 \times 10^{-10}\right)$. We also observed genome-wide significant association at $H M G 20 A\left(P=4.6 \times 10^{-9}\right)$ and $G R B 14\left(P=1.0 \times 10^{-8}\right)$, both implicated in a recent meta-analysis of T2D in South Asians ${ }^{10}$. Neither has previously been reported in European studies, and both remain genome-wide significant after removing PROMIS from the meta-analysis (HMG2OA P=1.9 $\left.\times 10^{-9} ; G R B 14 P=5.8 \times 10^{-9}\right)$. The lead SNPs from both meta-analyses are in strong linkage disequilibrium (LD) (HMG20A $r^{2}$ $=0.89$ and $G R B 14 r^{2}=0.77$ in CEU), and likely represent the same association signals. At the previously unreported loci, we observed nominal evidence of association $(P<0.05)$ in the South Asian ${ }^{10}$ and recent East Asian ${ }^{11}$ meta-analyses for the lead SNPs at $M C 4 R$ and ZMIZ1 (Supplementary Table 3), with consistent directions of effect across all three ancestry groups.

Several of these signals map to loci previously implicated in T2D-related metabolic traits (Supplementary Table 4). The lead SNP at $M C 4 R$ is in strong LD with variants associated with BMI ${ }^{14,15}\left(\mathrm{CEU} r^{2}=0.80\right)$ and triglycerides ${ }^{16}\left(\mathrm{CEU} r^{2}=0.84\right)$ and is associated with waist circumference and insulin resistance ${ }^{17}$. As with $F T O$, the T2D-effect at $M C 4 R$ is probably secondary to the BMI association. The lead SNP at GRB14 is highly correlated with variants associated with waist-hip ratio (WHR $)^{18}$ and high-density lipoprotein (HDL) cholesterol $^{16}\left(\mathrm{CEU} r^{2}=0.93\right)$. At $C I L P 2$, the lead SNP for T2D is also associated with triglycerides, low-density lipoprotein (LDL) and total cholesterol ${ }^{16}$. In contrast, the previously-reported association signals for haemoglobin $\mathrm{A} 1 \mathrm{C}\left(\mathrm{HbA}_{1 \mathrm{C}}\right)$ levels ${ }^{19}$ near $A N K 1$ 
are both independent $\left(\mathrm{CEU} r^{2}<0.01\right)$ of the lead T2D SNP from our meta-analysis. Given the role played by rare $A N K 1$ mutations in hereditary anemias, the $\mathrm{HbA}_{1 \mathrm{C}}$ associations at this locus were assumed to be driven by abnormal erythrocyte development and/or function. However, our newly discovered independent association with T2D (in cohorts where $\mathrm{HbA}_{1 \mathrm{C}}$ was not used for diagnosis) suggests that variation at this locus also has direct effects on glucose homeostasis.

\section{Insights into the genetic architecture of T2D}

The associated lead variants at the eight newly identified loci were common (Stage $2 \mathrm{RAF}$ 0.08-0.89) and had modest effects on T2D susceptibility (allelic odds ratios (OR) 1.07-1.14). Under a multiplicative model within and between variants, the sibling relative risk attributable to lead SNPs rose from $\lambda_{S}=1.093$ at the 55 previously described autosomal T2D loci represented on Metabochip ( $D U S P 9$ on chromosome $\mathrm{X}$ is not captured) to $\lambda_{\mathrm{S}}=$ 1.104 after inclusion of the eight newly discovered loci (Supplementary Table 5). Assuming a T2D population prevalence of $8 \%$, these 63 newly discovered and established autosomal loci together account for $5.7 \%$ of variance in disease susceptibility, as calculated by transforming dichotomous disease risk onto a continuous liability scale ${ }^{20}$ (Online Methods).

To determine the extent to which additional common variant associations contribute to the overall variance explained, we compared directional consistency in allelic effects between the two stages of the meta-analysis. Figure 1 presents the distribution of $Z$-scores from Stage 2, aligned to the risk allele from Stage 1, at a subset of 3,412 independent (CEU $r^{2}<0.05$ ) T2D replication variants that excludes lead SNPs and possible proxies (CEU $\left.r^{2} \geq 0.1\right)$ at the 63 newly discovered and established loci represented on Metabochip. The blue curve represents the expected distribution of Stage $2 Z$-scores under the null hypothesis of no association. There is a clear shift in the observed distribution, corresponding to closer agreement in the direction of allelic effect than expected by chance: $2,172(69.1 \%)$ of the 3,412 SNPs are concordant (binomial test $P=2.0 \times 10^{-104}$ ). For comparison, we examined T2D association patterns in 2,707 independent replication SNPs for QT-interval, the trait showing weakest correlation with T2D susceptibility among those contributing to Metabochip and found far less directional consistency (54.4\%, binomial test $P=3.3 \times 10^{-6}$ ). This modest enrichment most likely reflects weak overlap of risk alleles between the two traits, since exclusion of SNPs mapping within $300 \mathrm{~kb}$ of directionally consistent T2D replication variants reduced this excess $(52.5 \%$, binomial test $P=0.060)$.

The observed distribution of $Z$-scores can be considered a mixture of: (i) the "null distribution" of SNPs having no effect on T2D; and (ii) the "alternative distribution" of T2D-associated SNPs (Online Methods). We estimated the features of this alternative distribution (red curve) and noted that addition of this class of SNPs significantly improved the fit to the observed $Z$-scores over the null model. Using simulations, based on parameter estimates from this mixture model, we estimated that 488 (95\% confidence interval (CI) 456-521) of the independent replication SNPs, in addition to the 63 newly discovered and established loci, are associated with T2D susceptibility. For comparison, we undertook false-discovery rate (FDR) analysis of the 64,646 SNPs on the Metabochip selected for replication of any trait, using $P$-values from the combined meta-analysis (Online Methods). We observed broad agreement between combined meta-analysis $P$-values, FDR $Q$-values and the posterior probability of alternative distribution membership from the mixture model (Supplementary Fig. 4).

We were concerned that these additional, weaker association signals might reflect subtle stratification effects not eliminated by genomic control correction. However, using diverse European populations from the 1000 Genomes Project ${ }^{13}$ (Online Methods), we found no 
evidence that directionally-consistent T2D replication SNPs differed from other Metabochip replication SNPs with respect to $F_{S T}(P=0.88)$.

As expected, the estimated allelic ORs of the 488 SNPs are modest (1.01-1.11 in Stage 2), and larger samples would be required to establish association at genome-wide significance. For example, by simulating an additional 100,000 T2D cases and 100,000 controls as a "third stage" to the combined meta-analysis, we calculate that only $37 \%$ of the 488 replication SNPs in the alternative distribution would achieve this threshold. We estimate that these variants jointly account for $\lambda_{\mathrm{S}}=1.088$ (95\% CI 1.083-1.094), increasing the overall liability-scale variance explained to $10.7 \%$ (10.4-11.0\%).

\section{Additional sources of variation contributing to susceptibility}

These estimates likely set a lower bound to the overall liability-scale variance attributable to common SNPs. The mixture model does not take account loci not represented by Metabochip T2D replication SNPs due to failures in array design or manufacture or because the association signal in DIAGRAMv3 was too weak to merit inclusion. Indeed, the latter applied to two of the genome-wide significant loci, ANKRD55 and GRB14, which were nominated for inclusion on Metabochip because of associations with WHR (ANKRD55 and GRB14), blood pressure (ANKRD55) and plasma lipid concentrations (GRB14), rather than T2D.

To estimate the contribution to the variance explained by common variants genome-wide, we undertook polygenic mixed linear modelling analyses using GCTA ${ }^{21,22}$ in two DIAGRAMv3 GWAS data sets: DGI (1,022 cases, 1,075 controls) and WTCCC $(1,924$ cases, 2,938 controls). The estimated liability-scale variance explained by the full set of GWAS SNPs was consistent between the two studies: 62.6\% for DGI (95\% CI 38.1-87.1\%) and $63.9 \%$ for WTCCC (95\% CI 52.1-75.8\%). These results are similar to those obtained from a complementary method integrating polygenic risk score analysis and approximate Bayesian computation ${ }^{23}$ applied to the DIAGRAMv2 meta-analysis ${ }^{4}$, which estimated that $\sim 49 \%$ of liability-scale variance was explained by common variants genome-wide. These data indicate that a substantial proportion of the variation in T2D risk is captured by common variant association signals that, individually, lie beyond unequivocal detection in single SNP analyses.

The DIAGRAMv2 meta-analysis ${ }^{4}$ had provided some evidence for loci harboring multiple independent association signals. To understand the extent to which additional variance might be attributable to multiple variants at established and newly discovered loci, we extended these analyses, focusing on the detection of independent ( $\left.\mathrm{CEU} r^{2}<0.05\right)$ association signals that lie outside the recombination interval containing the lead SNP (Supplementary Table 2). We detected two loci at which multiple independent association signals attained genome-wide significance: $K C N Q 1$ (rs163184, $P=1.2 \times 10^{-11} ;$ rs $231361, P$ $\left.=1.2 \times 10^{-9} ; \mathrm{CEU} r^{2}=0.01\right)$ and $C D K N 2 A / B\left(\mathrm{rs} 10811661, P=3.7 \times 10^{-27} ; \mathrm{rs} 944801, P=\right.$ $2.4 \times 10^{-9}$; CEU $r^{2}=0.01$ ) (Fig. 2). Both signals at $K C N Q 1$ have previously been reported in East Asian and European populations ${ }^{4,24}$. However, the secondary signal at $C D K N 2 A / B$, which maps to the non-coding $C D K N 2 B-A S 1$ ( $A N R I L$ ) transcript, has not previously been implicated in T2D susceptibility. This signal is independent of the previously reported haplotype effect at the primary T2D signal at this locus, which is itself likely due to the phase relationships between two clades of partially correlated variants ${ }^{25,26}$. We also observed putative independent associations $\left(P<10^{-5}\right)$ at $D G K B($ rs $17168486, P=5.9 \times$ $10^{-11} ;$ rs6960043, $\left.P=3.4 \times 10^{-7} ; \mathrm{CEU} r^{2}=0.01\right)$ and $M C 4 R\left(\mathrm{rs} 12970134, P=1.2 \times 10^{-8}\right.$; rs11873305, $\left.P=3.8 \times 10^{-7} ; \mathrm{CEU} r^{2}=0.02\right)$. These results suggest that multiple independent association signals are widespread at T2D susceptibility loci. Imputation up to the more complete reference panels emerging from the 1000 Genomes Project ${ }^{13}$ and recently 
developed approaches that support approximate conditional analyses using meta-analysis summary level data ${ }^{27}$ will be important tools for documenting the full extent of such effects, especially where the variants map to the same recombination interval.

It has been argued that common variant association signals will often reflect unobserved causal alleles of lower frequency and greater effect size ${ }^{28}$. The fine-mapping content of Metabochip allowed us to seek empirical evidence to support this "synthetic association" hypothesis. We estimate, using 1000 Genomes Project data ${ }^{13}$ applied to HapMap CEU samples, that the array captures (CEU $\left.r^{2} \geq 0.8\right) 89.6 \%$ of common SNPs (minor allele frequency (MAF) $\geq 5 \%$ ) and $60.0 \%$ of low-frequency variants ( $1 \% \leq \mathrm{MAF}<5 \%)$ across Metabochip fine-mapping regions ${ }^{12}$. This represents a substantial improvement over HapMap ${ }^{29,30}$ which, across the same regions, captures $76.8 \%$ and $32.4 \%$ of common and low-frequency variants, respectively.

Across 36 fine-mapping regions on Metabochip that contain T2D susceptibility loci (including 27 explicitly chosen by DIAGRAM), we compared the characteristics of previously reported lead SNPs (defined by GWAS and HapMap imputation) and those emerging from the Stage 2 Metabochip meta-analysis. We restricted these comparisons to Stage 2 to avoid penalizing low-frequency variants not typed or well-imputed in Stage 1 . The GWAS and Metabochip lead SNPs were the same, or highly-correlated (CEU $r^{2}>0.8$ ), at 20 loci ( 15 with CEU $r^{2}>0.95$ ) (Supplementary Table 6). The low LD between GWAS and Metabochip lead SNPs at $D G K B$ and $K C N Q 1$ (both CEU $I^{2}=0.00$ ) arises because they "switch" between independent association signals at these loci (Fig. 2). For the remaining 14 loci, there was only modest LD between the previously reported GWAS and Metabochipdefined lead SNPs (CEU $r^{2}$ between 0.06 and 0.77). However, at only two loci did the lead SNP after Metabochip fine-mapping have substantially lower MAF and higher OR than the previously reported GWAS lead SNP: $P R O X 1$ (rs17712208, MAF $=0.03, \mathrm{OR}=1.20$; rs 340874, $\mathrm{MAF}=0.48, \mathrm{OR}=1.06)$ and $K L F 14(7-130116320, \mathrm{MAF}=0.02, \mathrm{OR}=1.10$; rs972283, $\mathrm{MAF}=0.48, \mathrm{OR}=1.01)$. Since coverage across Metabochip fine-mapping regions is incomplete, we cannot unequivocally exclude the presence of causal lowfrequency alleles at any single locus. However, the paucity of low-frequency candidate alleles across 36 loci suggests that most causal variants at these loci are common. A contribution of even rarer causal alleles (too rare to be represented on Metabochip) is also unlikely because the substantial effect sizes required to drive common variant association signals are inconsistent with the modest familial aggregation of $\mathrm{T} 2 \mathrm{D}^{23}$. This interpretation, favoring common causal alleles, is in agreement with the observed consistency of T2D risk variant associations across major ancestry groups ${ }^{31}$.

\section{Sex-differentiated analyses}

We performed sex-differentiated meta-analysis ${ }^{32}$ (Online Methods and Supplementary Figs. 5 and 6) to test for association of each SNP with T2D, allowing for heterogeneity in allelic effects between males (20,219 cases, 54,604 controls) and females (14,621 cases, 60,377 controls), thereby identifying two additional loci achieving genome-wide significance (Table 2 and Supplementary Table 7). The association signal mapping near $C C N D 2$ is most significant in males (male $P=1.1 \times 10^{-9}$, female $P=0.036$, heterogeneity $P=0.013$ ), while that upstream of GIPR is most significant in females (female $P=2.2 \times 10^{-7}$, male $P=$ 0.0037 , heterogeneity $P=0.057$ ) (Supplementary Fig. 7). The lead sex-differentiated SNP in GIPR is only weakly correlated with previously reported associations with BMI ${ }^{15}\left(\mathrm{CEU} r^{2}=\right.$ $0.06)$ and two-hour glucose levels ${ }^{33}\left(\mathrm{CEU} r^{2}=0.07\right)$ (Supplementary Table 4).

The sex-differentiated analyses also revealed nominal evidence of heterogeneity $(P<0.05)$ at four established T2D susceptibility loci (Table 2 and Supplementary Tables 7 and 8): $K C N Q 1(P=0.0013), D G K B(P=0.0068)$ and BCL11A $(P=0.012)$ were most 
significantly associated in males, and $G R B 14(P=0.0080)$ in females. The sexdifferentiated association at $G R B 14$ is consistent with the female-specific effect on WHR observed at this locus ${ }^{18}$. As $K C N Q 1$ and $D G K B$ demonstrate multiple independent associations in the sex-combined meta-analysis, we investigated whether sex differences in allelic effects were consistent across these signals (Supplementary Fig. 8). This appeared true for $D G K B$ (rs17168486, male $P=6.5 \times 10^{-13}$, female $P=0.0052$; rs6960043, male $P=$ $7.9 \times 10^{-7}$, female $P=0.015$ ), but not KCNQ1 (rs 163184, male $P=8.5 \times 10^{-15}$, female $P=$ $7.8 \times 10^{-3} ;$ rs 231361 , male $P=2.9 \times 10^{-6}$, female $P=2.9 \times 10^{-6}$ ).

\section{Understanding the biology of T2D susceptibility loci}

For most T2D susceptibility loci, the underlying causal variants and the genes through which they act are yet to be identified, and the pathophysiological processes mediating disease risk remain unclear. We applied a variety of approaches to the newly discovered and established T2D susceptibility loci, and in some cases to putative loci with more modest evidence of association, to identify mechanisms involved in disease pathogenesis.

\section{Physiological analyses}

As noted earlier, lead SNPs at several newly identified loci are in strong LD with variants associated with other T2D-related metabolic traits. To gain a more complete picture of patterns of trait overlap, we first assessed the effect of T2D risk alleles on glycemic traits in European-descent meta-analyses from the MAGIC Investigators (Online Methods). Fasting glucose associations were analyzed for up to 133,010 non-diabetic individuals with GWAS and/or Metabochip data ${ }^{34}$. In addition to the nine loci previously reported (MTNR1B, DGKB, ADCY5, PROX1, GCK, GCKR, TCF7L2, SLC3OA8 and C2CD4A $)^{4,5}$, four more T2D association signals were genome-wide significant for fasting glucose: $C D K N 2 A / B(P=$ $\left.5.7 \times 10^{-18}\right), A R A P 1\left(P=1.2 \times 10^{-10}\right), \operatorname{IGF} 2 B P 2\left(P=1.8 \times 10^{-8}\right)$ and $C D K A L 1(P=2.0 \times$ $10^{-8}$ ) (Supplementary Table 9). The $Z B E D 3$ locus also attained genome-wide significance with fasting glucose after adjustment for BMI $\left(P=1.2 \times 10^{-8}\right)$. In contrast, lead T2D SNPs at 27 of the newly discovered and established loci showed no evidence of association with fasting glucose $(P>0.05)$, despite sample sizes ranging from 38,424 to 132,999 individuals (Supplementary Table 10 and Supplementary Fig. 9). Lead T2D SNPs at the remaining 24 loci were nominally associated with fasting glucose $(P<0.05)$, all with directionally consistent effects. These data extend previous reports indicating that the genetic landscape of pathological and physiological variation in glycemia is only partially overlapping, and are consistent with reciprocal analyses reported in the companion MAGIC paper $^{34}$.

Second, we extended our previous analysis ${ }^{4}$ of the physiological consequences of T2D risk alleles to include the newly identified loci. We used the published MAGIC meta-analysis (up to 37,037 non-diabetic individuals) of HOMA indices of beta-cell function and insulin sensitivity ${ }^{5}$ as these traits were not included in the enlarged Metabochip study ${ }^{34}$. The risk allele at $A N K 1$ has features (nominally significant reduction in HOMA-B) indicating a primary effect on beta-cell function, whereas those at GRB14 and AKNRD55 are characteristic of loci acting primarily through insulin resistance (increased HOMA-IR) (Supplementary Fig. 10 and Supplementary Table 10). The results for GRB14 are consistent with its broad impact on insulin-resistance related traits (described below), while at $A K N R D 55$, these analyses point to MAP3K1, encoding MEK kinase, a key component of the insulin-signalling pathway, as the stand-out local candidate.

Next, we examined the effect of T2D risk alleles on anthropometric and lipid traits using data from the GIANT Consortium (up to 119,600 individuals after excluding data from T2D case series) ${ }^{15}$ and the Global Lipids Genetics Consortium (up to 100,184 individuals) ${ }^{16}$ (Online Methods and Supplementary Tables 11 and 12). The only lead SNP to demonstrate 
convincing evidence of association $\left(P<10^{-5}\right)$ with adiposity was at $M C 4 R$. The lead SNPs at $M C 4 R$ and $G R B 14$ show the same pattern of lipid associations $\left(P<10^{-5}\right)$ : reduced HDL and raised triglycerides. In contrast, the lipid associations at $C I L P 2$ and $G I P R$ ran counter to expected epidemiological correlations: T2D risk alleles were associated with reduced triglyceride levels at both loci, and at CILP2 with reduced LDL and total cholesterol.

Finally, we noticed that the lead T2D SNP at the BCAR1 locus is genome-wide significant for type 1 diabetes (T1D) ${ }^{35}$, although risk is conferred by the opposite alleles. Across 37 T1D susceptibility loci (Supplementary Fig. 11), we observed nominal evidence $(P<0.05)$ of association to T2D at six. For three of these (BCAR1, GLIS3 and RAD51L1), the T1D risk allele was protective for T2D, while at the others (C6orf173, COBL and C10orf59), the effects were coincident. These data indicate that rates of diagnostic misclassification among T2D cases in our study are low, and also highlight interesting points of overlap in the processes involved in risk of, and protection from, these two major forms of diabetes.

\section{Mapping potential causal transcripts and variants}

The T2D-association signals emerging from the present meta-analysis map to regions containing many transcripts and potential functional variants. To identify promising regional transcripts, we examined expression quantitative trait locus (eQTL) data from a variety of tissues (Online Methods and Supplementary Note). At six of the newly discovered loci, the lead T2D SNP showed strong cis-eQTL associations and was highly correlated (CEU $r^{2}>$ 0.8 ) with the lead cis-eQTL SNP (Supplementary Table 13). These "coincident" eQTL implicate GRB14 (omental fat), $A N K 1$ (omental and subcutaneous fat, liver and prefrontal cortex), KLHDC5 (blood, T cells and CD4+ lymphocytes), BCAR1 (blood), ATP13A1 (at the CILP2 locus, blood and monocytes), HMG2OA (liver) and LINGO1 (also at the $H M G 20 A$ locus, adipose tissue). For those loci (GRB14, ANK1 and BCAR1) for which individual-level expression data for the appropriate tissues were available ${ }^{36}$, we confirmed signal coincidence by conditional analyses (Online Methods and Supplementary Table 14).

We used 1000 Genomes Project data ${ }^{13}$ to search for non-synonymous variants in strong LD (CEU $r^{2}>0.8$ ) with lead SNPs at the newly discovered loci (Online Methods). The only candidate allele uncovered was a non-synonymous variant in exon 6 of TM6SF2 (19-19379549, CEU $r^{2}=0.98$ with rs 10401969) at the CILP2 locus. This change is predicted by SIFT $^{37}$ to have no appreciable effect on protein function.

\section{Pathway and protein-protein interaction analyses}

To extend previous efforts to define pathways and networks involved in T2D pathogenesis ${ }^{4}$, we combined meta-analysis data with protein-protein interactions (PPI), semantic relationships within the published literature and annotated pathways (Fig. 3). For these analyses, we generated a "primary" list of 77 transcripts mapping nearest to lead SNPs at T2D susceptibility loci or implicated in monogenic diabetes ${ }^{38}$ (Online Methods and Supplementary Table 15).

Using a refined database of high-confidence PPI ${ }^{39,40}$, we constructed a network of 314 proteins from these 77 transcripts using DAPPLE ${ }^{41}$. We detected an excess of physical interactions in the network, both direct (between the associated transcripts themselves, $P<$ $10^{-4}$ ) and indirect (via 237 shared interactors not on the list of associated transcripts, $P=$ 0.0070). There was no evidence that this set of shared interactors was enriched for T2Dassociated variants. Some interactions, such as those between the potassium channel encoding genes $K C N J 11$ and $A B C C 8$, are expected, while other sub-networks are of greater novelty. For example, the transcriptional co-activator protein $C R E B B P$, implicated in the coupling of chromatin remodelling to transcription factor recognition, does not map to any 
T2D susceptibility locus. However, it is the most connected gene for protein-level interactions $(P<0.005)$ in the PPI network, interacting with nine primary transcripts, eight implicated in monogenic diabetes or mapping to established T2D susceptibility loci (HNF1A, HNF1B, HNF4A, PLAGL1, TCF7L2, PPARG, PROX1 and NOTCH2) and one from a locus with a strong, but not genome-wide significant, association (ETS1, lead SNP rs7931302, $\left.P=3.8 \times 10^{-7}\right)$. Other shared interactors identified through these analyses included SERTAD1, FOXO1, PPARGC1A, GRB10 and MAFA. Several of these play roles in the transcriptional regulation of diabetes-relevant tissues, and some also interact with $C R E B B P$. We used a pre-defined set of 1,814 genes encoding "DNA-binding proteins" (Online Methods) to show that: (i) T2D signals are highly enriched for transcription factors (21 of 71 primary transcripts listed within the HGNC catalog, compared to 1,793 of 19,162, $P=2.3 \times 10^{-6}$ ); and (ii) transcription factors within T2D loci are enriched for interaction with $C R E B B P$ (taking the 1,164 listed in the protein interaction database, 9 of 21 compared with 127 of 1,143, $P=2.7 \times 10^{-4}$ ). These data suggest that modulation of CREBBP-binding transcription factors plays an important role in T2D susceptibility.

The same set of 77 primary transcripts showed modest evidence of excess connectivity $(P=$ 0.020 by permutation) using text-mining approaches ${ }^{42}$ (Online Methods). When we used this set of 77 genes as a "seed" to query a list of 77 "secondary" transcripts (nearest to lead SNPs with posterior probability of T2D-association $>75 \%$ from the mixture model) (Supplementary Table 15), we found significant connections $(P<0.001)$ between the primary associated transcripts and four other genes: $L E P R$ (leptin obesity pathways), $M Y C$ (cell-cycle pathway), GATA6 (pancreas development pathway) and DLL4 (Notch signalling target).

We also tested for enrichment of GWAS associated transcripts in pathway data. To retain power, we focused on 16 biological hypotheses chosen for assumed relevance to T2D pathogenesis, 43-45 (Supplementary Note). We used a two-step modified gene-set enrichment analysis (GSEA) approach applied sequentially to Stage 1 (using MAGENTA ${ }^{46}$ ) and Stage 2 meta-analyses (Online Methods and Supplementary Table 16). Of the 16 biological hypotheses tested, two demonstrated reproducible enrichment of T2D associations. The strongest enrichment was observed for a broader set of primary and secondary transcripts mapping to T2D-associated loci in the adipocytokine signalling pathway (MAGENTA $P=6.2 \times 10^{-5}$; modified GSEA $P=1.6 \times 10^{-4}$ ). This gene set includes the adiponectin, leptin and TNF-alpha signalling pathways previously implicated in the development of insulin resistance ${ }^{47}$, but for which genome-wide significant common variant associations with T2D susceptibility have not been previously reported. This analysis highlighted eight genes in this pathway most likely to be causal for T2D susceptibility:

IRS1, LEPR, RELA, RXRG, ACSL1, NFKB1, CAMKK1 and a monogenic diabetes gene $A K T 2$. Members of this pathway were also strongly represented (17 out of 314) in the DAPPLE PPI network $\left(P=7.5 \times 10^{-14}\right)$. Modest but robust enrichment was also observed for genes influencing cell cycle, in particular regulators of the G1 phase during mitosis (MAGENTA $P=2.0 \times 10^{-4}$; modified GSEA $P=3.0 \times 10^{-3}$ ). The majority of genes driving these cell-cycle enrichments were cyclin-dependent kinase (CDK) inhibitors (CDKN2A/B, $C D K N 1 C$ and $C D K N 2 C$ ) and cyclins that activate CDKs (CCNE2, CCND2 and CCNA2). Many of these regulate $C D K 4$ or $C D K 6$, which are known to play a role in pancreatic betacell proliferation ${ }^{48,49}$. We saw no evidence of enrichment for other processes implicated in T2D pathogenesis, including amyloid formation, ER stress and insulin signalling.

\section{DISCUSSION}

We have expanded T2D association analysis to almost 150,000 individuals. In so doing, we have added another 10 loci to the list of confirmed common variant signals: for several of 
these, we have identified strong positional candidates based on expression data and known biology. The data support the view that much of the overall variance in T2D susceptibility can be attributed to the impact of a large number of common causal variants, most of very modest effect. While such a model poses challenges for accumulating genome-wide significant evidence of association at a specific variant, it does suggest that genetic profiling based on the entirety of sequence variation has the potential to provide useful risk stratification for T2D.

If common causal alleles explain a substantial component of T2D susceptibility, the contribution of rare and low-frequency risk variants may be less than is often assumed: resequencing studies will soon provide empirical data to address this question. In particular, it will be important to determine whether, as the number of susceptibility loci increases, there is evidence that the pathophysiological mechanisms implicated by human genetics coalesce around a limited set of core pathways and networks. Our data suggest that this may be the case, with a variety of analytical approaches pointing to cell cycle regulation, adipocytokine signalling and CREBBP-related transcription factor activity as key processes involved in T2D pathogenesis.

\section{ONLINE METHODS}

\section{Stage 1 meta-analysis}

The Stage 1 meta-analysis consisted of 12,171 T2D cases and 56,862 controls across 12 GWAS from European descent populations (Supplementary Table 1). Samples were typed with a range of GWAS genotyping products. Sample and SNP quality control (QC) were undertaken within each study. Each GWAS was then imputed at up to 2.5 million SNPs using CEU samples from Phase II of the International HapMap Project ${ }^{28}$. Each SNP with MAF $>1 \%$ passing QC was tested for association with T2D under an additive model after adjustment for study-specific covariates, including indicators of population structure. The results of each GWAS were corrected for residual population structure using the genomic control inflation factor ${ }^{50}$ and were combined via fixed-effects inverse-variance weighted meta-analysis. The results of the Stage 1 meta-analysis were subsequently corrected by genomic control $\left(\lambda_{\mathrm{GC}}=1.10\right)$.

\section{Stage 2 meta-analysis}

The Stage 2 meta-analysis consisted of 21,491 T2D cases and 55,647 controls across 25 studies from European descent populations and 1,178 T2D cases and 2,472 controls from one study of Pakistani descent (PROMIS) (Supplementary Table 1). All samples were genotyped with Metabochip. Sample and SNP QC were undertaken within each study. Each SNP with MAF $>1 \%$ passing QC was tested for association with T2D under an additive model after adjustment for study-specific covariates. We would expect inflation in association signals across the content of Metabochip, even in the absence of population structure, because it has been designed to be enriched for T2D and other T2D-related metabolic trait loci. The results of each study were thus corrected for residual population structure using the genomic control inflation factor obtained from a subset of 3,598 independent "QT-interval" SNPs (CEU $\left.I^{2}<0.05\right)$, which were not expected to be associated with T2D. The Stage 2 meta-analysis was performed in two steps: (i) combine all studies of European descent; and (ii) add the PROMIS study. In both steps, the results of each study were combined via fixed-effects inverse-variance weighted meta-analysis. The results of the Stage 2 European meta-analysis were corrected by "QT-interval" genomic control $\left(\lambda_{\mathrm{QT}}=\right.$ 1.19), but this adjustment was not then necessary after the addition of PROMIS $\left(\lambda_{\mathrm{QT}}=0.99\right.$ was less than 1). Heterogeneity in allelic effects between European descent studies and 
subsequently between the European meta-analysis and PROMIS was assessed by means of Cochran's $Q$-statistic 51 .

\section{Combined meta-analysis}

The results of the Stage 1 and Stage 2 meta-analyses were combined for all Metabochip SNPs via fixed-effects inverse-variance weighted meta-analysis. The combined metaanalysis consisted of 34,840 cases and 114,981 controls. This was performed in two steps: (i) combine Stage 1 meta-analysis with European descent Stage 2 meta-analysis; and (ii) add the PROMIS study. The results of the combined European meta-analysis was corrected by "QT-interval" genomic control $\left(\lambda_{\mathrm{QT}}=1.13\right)$, but this adjustment was not necessary after the addition of PROMIS ( $\lambda_{\mathrm{QT}}=0.98$ was less than 1) (Supplementary Fig. 12). Heterogeneity in allelic effects between the Stage 1 and Stage 2 meta-analyses was assessed by means of Cochran's $Q$-statistic.

\section{Look-up of meta-analysis results for lead SNPs in GWAS of South and East Asian descent}

We obtained summary statistics (RAFs, association $P$-values, allelic ORs and $95 \%$ CIs) for lead SNPs at the newly discovered loci in meta-analyses of T2D GWAS in: (i) 5,561 cases and 14,458 controls of South Asian descent ${ }^{10}$, excluding 1,958 overlapping samples from PROMIS that were also included in our study, comprising 568,976 directly genotyped autosomal SNPs; and (ii) 6,952 cases and 11,865 controls of East Asian descent ${ }^{11}$, comprising 2,626,356 directly genotyped and imputed autosomal SNPs. For each SNP, summary statistics were aligned to the risk allele in our primarily European descent metaanalysis.

\section{Calculation of sibling relative risk and liability-scale variance explained}

Assuming a multiplicative model (within and between variants), the contribution to the sibling relative risk of a set of NSNPs is given by

$$
\lambda_{s}=\prod_{j=1}^{N}\left[1+\frac{p_{j}\left(1-p_{j}\right)\left(\psi_{j}-1\right)^{2}}{2\left[\left(1-p_{j}\right)+p_{j} \psi_{j}\right]^{2}}\right]^{2}
$$

where $p_{j}$ and $\psi_{j}$ denote the RAF and corresponding allelic OR at the $j$ th $\mathrm{SNP}^{52}$. Assuming disease prevalence, $K$, the liability-scale variance ${ }^{20}$ explained by these SNPs is given by

$$
h_{L}^{2}=\frac{2\left[T-T_{1} \sqrt{\left(1-\left(T^{2}-T_{1}^{2}\right)(1-T / \omega)\right]}\right)}{\omega+T_{1}^{2}(\omega-T)}
$$

In this expression, $T=\varphi^{-1}(1-K), T_{1}=\varphi^{-1}\left(1-\lambda_{S} K\right)$, and $\omega=Z / K$, where $z$ is the height of the standard Gaussian density at $T$.

\section{Z-score mixture modelling}

We considered the distribution of $Z$-scores from the Stage 2 meta-analysis, aligned to the risk allele from Stage 1, at a subset of 3,412 independent T2D replication variants (CEU $r^{2}<$ $0.05)$, excluding lead SNPs and proxies (CEU $r^{2} \geq 0.1$ ) at the 63 established and newly discovered susceptibility loci on Metabochip. The Stage $2 Z$-scores were modelled as a mixture of two Gaussian distributions: (i) with mean zero and unit variance (i.e. under the null hypothesis of no association); and (ii) with unknown mean (greater than zero) and variance (i.e. under the alternative hypothesis). The mean and variance of the alternative 
distribution, and the mixing proportion, were estimated using an expectation-maximization algorithm.

We estimated the posterior probability that each of the 3,412 independent replication SNPs is truly associated with T2D from the mixture distribution. We approximated the contribution of these SNPs to $\lambda_{S}$ by simulation from the mixture distribution. For each simulated replicate, we selected "causal" variants at random from these SNPs according to their posterior probability of association. Over 1,000 replicates, we approximated the mean and 95\% CI for: (i) the number of "causal" variants among the 3,412 independent replication SNPs; and (ii) the contribution to $\lambda_{\mathrm{S}}$, using estimated RAFs and allelic ORs from the Stage 2 meta-analysis. For each replicate, we also generated a hypothetical third stage to the study consisting of 100,000 T2D cases and 100,000 controls. For each "causal" variant, we generated association summary statistics ( $Z$-score aligned to the risk allele from Stage 1$)$ according to the RAF and allelic OR from our Stage 2 meta-analysis.

\section{Assessment of allele frequency variation across European populations}

We calculated F-statistics $\left(F_{S T}\right)$ across European populations using data from the 1000 Genomes Project (CEU, TSI, FIN, GBR and IBS) ${ }^{13}$ for the subset of SNPs selected for replication on Metabochip. $F_{S T}$ was calculated by comparing mean heterozygosity across all populations to the mean within each sub-population, weighted by the number of contributing chromosomes from each sub-population. We compared $F_{S T}$ for the subset of T2D replication SNPs that were directionally consistent between Stage 1 and Stage 2 metaanalyses with all Metabochip replication SNPs (up to 65,345 SNPs), using the KolmogorovSmirnov test.

\section{False-discovery rate (FDR) analysis}

We undertook FDR analysis ${ }^{53}$ of 64,646 Metabochip replication SNPs using combined meta-analysis $P$-values. From this analysis, we observed $\widehat{\pi}_{0}=0.88$, consistent with an excess of true positives in this set. We compared these $P$-values with FDR $Q$-values and posterior probabilities of membership to the alternative distribution from the mixture model (Supplementary Fig. 4) at the set of 2,172 T2D replication SNPs with concordant direct of allelic effect in both stages of the meta-analysis, after exclusion of 11 AT/GC SNPs with obvious strand orientation misalignments. FDR analysis also indicated an excess of expected true positives in this set of SNPs, even at relatively consistent thresholds (for example, we expect one false positive and 66 true positives at a $Q$-value of 0.014 ).

\section{Sex-differentiated meta-analysis}

The Stage 1, Stage 2 and combined meta-analyses described above were repeated for males and females separately with correction for population structure within each sex (Supplementary Fig. 13). The male-specific meta-analysis consisted of 20,219 cases and 54,604 controls, while the female-specific meta-analysis consisted of 14,621 cases and 60,377 controls. The sex-specific meta-analyses were then combined to conduct a sexdifferentiated test of association and a test of heterogeneity in allelic effects between males and females ${ }^{32}$.

\section{Physiological analyses}

We obtained summary statistics (association $P$-values and $Z$-scores for direction of effect or allelic effects and standard errors) for lead T2D SNPs in GWAS meta-analyses of metabolic traits in European descent populations. Summary statistics were aligned to the T2D risk allele from the combined meta-analysis. We obtained summary statistics for lead SNPs in all newly discovered and established loci for glycemic traits in non-diabetic individuals from 
the MAGIC Investigators ${ }^{5,34}$. For fasting glucose and fasting insulin, the meta-analysis comprised up to 133,010 individuals, genotyped with GWAS arrays and imputed on up to $\sim 2.5$ million SNPs, or genotyped with Metabochip. We also considered surrogate estimates of beta-cell function (HOMA-B) and insulin resistance (HOMA-IR) derived by homeostasis model assessment in up to 38,238 individuals (from GWAS meta-analysis only since these traits were not investigated in the enlarged MAGIC Metabochip study). We obtained summary statistics for lead SNPs in the newly discovered T2D loci (also including GRB14 and $H M G 20 A$ ) for BMI in up to 119,600 individuals from the GIANT Consortium ${ }^{15}$. To eliminate potential bias in BMI allelic effect estimates at T2D susceptibility loci ${ }^{54}$, we restricted our attention to meta-analysis of population-based studies not ascertained for disease status for $\sim 2.8$ million directly genotyped and/or imputed SNPs. We obtained summary statistics for the same SNPs for plasma lipid concentrations from the Global Lipids Genetics Consortium ${ }^{16}$. This meta-analysis comprised $\sim 2.6$ million directly genotyped and/ or imputed SNPs assessed for association to plasma concentrations of: total cholesterol (up to 100,184 individuals); LDL (up to 95,454 individuals); HDL (up to 99,900 individuals); and triglycerides (up to 96,598 individuals).

We also examined T2D association summary statistics at lead SNPs for 37 established T1D susceptibility loci. For each of these SNPs, we reported the allelic OR (aligned to the T2D risk-allele) and $P$-values in: (i) our Stage 1 T2D meta-analysis; and (ii) a GWAS metaanalysis of 7,514 T1D cases and 9,045 population controls from European descent populations from the Type 1 Diabetes Genetics Consortium ${ }^{35}$.

\section{Expression analyses}

We identified proxies (CEU $r^{2}>0.8$ ) for each lead T2D SNP in our newly discovered loci (also including GRB14 and HMG20A). We interrogated public databases and unpublished resources for cis-eQTL expression with these SNPs in multiple tissues (details of these resources are summarized in the Supplementary Note). The collated results from these resources met study-specific criteria for statistical significance for association with transcript expression. For each transcript associated with a lead T2D SNP (or proxy), we identified the lead cis-eQTL SNP, and then estimated LD between them using 1000 Genomes Project data to assess coincidence of the signals.

We subsequently tested for association of each lead T2D SNP with the expression of flanking transcripts (within a $1 \mathrm{Mb}$ window) in 603 subcutaneous adipose tissue samples and 745 peripheral blood samples from individuals from the Icelandic population, genotyped using the Illumina HumanHap 300 Bead Array, and imputed up to $2.5 \mathrm{M} \mathrm{SNPs}{ }^{36}$. We modelled the log-average expression ratio of two fluorphores as a function of the allele count (expected allele count for imputed SNPs) in a linear regression framework, with adjustment for age and sex (and differential cell count for blood samples) as covariates. All $P$-values were also adjusted for the relatedness between individuals by simulating genotypes through the corresponding Icelandic genealogy ${ }^{55}$. We also identified the most strongly associated cis-eQTL SNP for each flanking transcript. We then performed a conditional test of association of the transcript with the cis-eQTL SNP within the same linear regression framework, with additional adjustment for the lead T2D SNP as a covariate. The conditional analyses determine whether the cis-eQTL SNP association with the transcript can be explained by the lead T2D SNP.

We searched the 1000 Genomes Project data (Phase I interim release) for non-synonymous variants in strong LD $\left(\mathrm{CEU} r^{2}>0.8\right)$ with lead T2D SNPs in the newly discovered loci (also including $G R B 14$ and $H M G 20 A$ ). Identified non-synonymous variants were subsequently interrogated for likely downstream functional consequences using SIFT $^{37}$. 


\section{Pathway, text mining and PPI analyses}

We generated two lists of transcripts on the basis of the results of the sex-combined and sexdifferentiated meta-analyses. The "primary" list included: (i) the nearest transcript to the lead SNP at 41 previously reported common variant loci identified in European descent populations; (ii) the nearest transcript to the lead SNP at the ten newly identified loci $(P<5$ $\times 10^{-8}$ ) from the sex-combined meta-analysis, including $G R B 14$ and $H M G 20 A$; (iii) the nearest transcript to the lead SNP at both novel signals $\left(P<5 \times 10^{-8}\right)$ from the sexdifferentiated meta-analysis; (iv) the nearest transcript to the lead SNP at six additional loci with the strongest evidence of association $\left(P<5 \times 10^{-7}\right)$ from the sex-combined metaanalysis; and (v) 18 genes implicated in monogenic forms of diabetes ${ }^{38}$, not already overlapping other loci included in the list. The "secondary" list incorporated the nearest transcript to the lead SNP at 77 additional loci with posterior probability of association of at least $75 \%$ from the mixture model, not already included in the primary list.

We tested the hypothesis that a PPI network built from the 77 primary transcripts was significantly enriched for physical interaction over and above that expected by chance using DAPPLE $^{41}$. To build networks, DAPPLE uses a refined database of high-confidence interactions ${ }^{39,40}$, which emphasizes confidence of interaction over completeness, with the result that not all proteins are represented. We considered two categories of interactions: direct (i.e. between the associated transcripts themselves) and indirect (i.e. via common interactors that were not among the associated transcripts). We assessed the significance of the enrichment of physical interactions by permutation. Subsequently, we used the network as a "seeds" to query against the 77 secondary transcripts.

We used GRAIL to highlight genes from T2D susceptibility loci using similarity of text in PubMed abstracts or in gene-ontology associated codes ${ }^{42}$. To reduce confounding by published T2D GWAS analyses, we restricted our analysis to abstracts published prior to December 2006. We first tested for enrichment of connectivity in the list of 77 primary transcripts (treating the 18 monogenic loci as a single locus to reduce confounding), and assessed significance via permutation ${ }^{4}$. These gene sets were then used as the "seed" against which the list of 77 secondary transcripts was queried for connectivity.

We employed a two-step GSEA strategy to test for enrichment of transcripts in T2D susceptibility loci within pathways pertaining to 16 biological hypotheses related to disease pathogenesis (full details of these hypotheses are presented in the Supplementary Note). In the first step, we applied MAGENTA ${ }^{46}$ to the Stage 1 meta-analysis. Genes in each pathway were scored on the basis of the most significant "local" SNP association using $-110 \mathrm{~kb} /+40$ $\mathrm{kb}$ boundaries. The $95^{\text {th }}$ percentile of association $P$-values from all genes in the genome was used to determine the enrichment cut-off. In the second "replication" step, nominally significant gene sets from step one (MAGENTA $P<0.05$ ) were tested for enrichment of T2D association signals in the Stage 2 meta-analysis. To account for the bias in the Metabochip design to SNPs nominally associated with T2D and related metabolic traits, we employed a modified GSEA approach. We tested for enrichment among a broader set of primary or primary and secondary transcripts within LD regions defined by $r^{2}>0.5$ on either side of the lead SNP, extended to the nearest recombination hotspot and then an additional $50 \mathrm{~kb}$ (if there was no gene within the LD region, we used the nearest transcript). For robustness testing, we also examined enrichment in the nearest gene to the lead SNPs. The modified GSEA $P$-value was computed as the fraction of randomly sampled sets of loci, matched for number and local gene density to our primary and secondary lists, which have the same or more significant hyper-geometric probability than that of the T2D loci. For the "null" set, we used 1,600 LD-pruned Metabochip T2D replication SNPs with the lowest posterior probability of association $(<5 \%)$ from the mixture model. To control for potential confounders, we applied the modified GSEA approach to two negative control lists: (i) loci 
defined by the lowest ranked independent T2D replication SNPs from our Stage 2 metaanalysis; and (ii) loci for QT-interval on the basis of our Stage 2 meta-analysis for independent replication SNPs for this trait, excluding those within our primary and secondary lists of T2D susceptibility loci and those near monogenic diabetes genes.

\section{Supplementary Material}

Refer to Web version on PubMed Central for supplementary material.

\section{Acknowledgments}

Funding for this study was provided by: Academy of Finland $(77299,102318,110413,118065,123885,124243$, 129680, 129293, 129494, 136895, 139635, 141005, 213506, 251217); Agence Nationale de la Recherche (France); American Diabetes Association (7-08-MN-OK); Association Française des Diabétiques; Association de Langue Française pour l'Etude du Diabète et des Maladies Métaboliques (France); Association Diabète Risque Vasculaire (France); BDA Research (UK); British Heart Foundation (RG/98002; RG2008/08); Cancer Research UK; Central Norway Health Authority; Central Finland Hospital District; Center for Inherited Disease Research (CIDR) (USA) ; Chief Scientist Office, Scotland (CZB/4/672); City of Kuopio (Finland); City of Leutkirch (Germany); Dept of Health (UK); Deutsche Forschungsgemeinschaft (ER1 55/6-2); Diabetes UK; Doris Duke Charitable Foundation (USA); Estonian Government SF0180142s0; European Commission: ENGAGE (HEALTH-F4-2007-201413); EXGENESIS (LSHM-CT-2004-005272); 245536; QLG1-CT-2002-00896; 2004310); European Commission (Marie Curie: FP7-PEOPLE-2010-IEF); European Regional Development Fund; Faculty of Medicine, Norwegian University of Science and Technology; Finnish Diabetes Association; Finnish Diabetes Research Foundation; Finnish Foundation for Cardiovascular Research; Finnish Heart Association; Finnish Medical Society; Folkhälsan Research Foundation (Finland); Food Standards Agency (UK); Foundation for Life and Health in Finland; Federal Ministry of Education and Research (BMBF) (Germany); Federal Ministry of Health (Germany); General Secretary of Research and Technology (Greece); German Center for Diabetes Research (DZD); German Research Council (GRK 1041); Great Wine Estates of the Margaret River region of Western Australia; Groupe d'Etude des Maladies Métaboliques et Systémiques (France); Harvard Medical School (USA); Heinz Nixdorf Foundation (Germany); Helmholtz Zentrum München-Research Center for Environment and Health (Germany); Helsinki University Central Hospital Research Foundation (Finland); IngaBritt and Arne Lundberg's Research Foundation (Sweden) (grant nr. 359); Ministry of Health (Ricerca Corrente) (Italy); Karolinska Institutet (Sweden); Knut and Alice Wallenberg Foundation (Sweden) (KAW 2009.0243); Kuopio University Hospital (Finland); Municipal Heath Care Center and Hospital, Jakobstad, Finland; Ministry of Social Affairs and Health (Finland); Ministry of Education and Culture (Finland) (627;2004-2011); Ministry of Innovation, Science, Research and Technology of the state North Rhine-Westphalia (Germany); Medical Research Council (UK) (G0000649,G0601261); MRC-GSK pilot programme grant (UK); Munich Center of Health Sciences (MC Health) (Germany); National Genome Research Network (NGFN) (Germany); NHLBI (HHSN268201100005C, HHSN268201100006C, HHSN268201100007C, HHSN268201100008C, HHSN268201100009C, HHSN268201100010C, HHSN268201100011C,

HHSN268201100012C, R01HL087641, R01HL59367, R01HL086694, N01-HC-25195, N02-HL-6-4278); NHGRI (U01HG004402, N01-HG-65403); National Institutes for Health (USA) (HHSN268200625226C, UL1RR025005, U01HG004399, 1R21NS064908, 1Z01-HG000024, AG028555, AG08724, AG04563, AG10175, AG08861, CA055075); NIDDK (DK062370, DK058845, DK072193, DK078616, DK080140, DK073490); Närpes Health Care Foundation (Finland); National Health Screening Service of Norway; National Institute of Health Research (UK); National Institute for Health and Welfare (Finland) ; Nord-Trøndelag County Council (Norway); Nordic Center of Excellence in Disease Genetics; Norwegian Institute of Public Health; Norwegian Research Council; Novo Nordisk Fonden (Denmark); Ollqvist Foundation (Sweden); Oxford NIHR Biomedical Research Centre (UK); Paavo Nurmi Foundation (Finland); Päivikki and Sakari Sohlberg Foundation (Finland); Perklén Foundation (Sweden); Pfizer; Pirkanmaa Hospital District (Finland); Programme National de Recherche sur le Diabéte (France); Programme Hospitalier de Recherche Clinique (French Ministry of Health); Region of Nord Pas De Calais (Contrat de Projets état-Région) (France); Research into Ageing (UK); Robert Dawson Evans Endowment of the Department of Medicine at Boston University School of Medicine and Boston Medical Center; Royal Swedish Academy of Sciences; Sarstedt AG \& Co. (Germany); Signe and Ane Gyllenberg Foundation (Sweden); Slottery Machine Association (Finland); Social Insurance Institution of Finland (4/26/2010); South OstroBothnia Hospital District (Finland); State of Baden-Württemberg, Germany; Stockholm County Council (560183, 562183; Stroke Association (UK); Swedish Research Council (8691;09533; 2009-1039; Dnr 521-2010-3490, Dnr 521-2007-4037, Dnr 521-2008-2974, Dnr 825-2010-5983; Dnr 349-2008-6589); Swedish Cultural Foundation in Finland; Swedish Diabetes Foundation; Swedish Heart-Lung Foundation; Swedish Foundation for Strategic Research; Swedish Society of Medicine; Swedish Research Council; Swedish Research Council for Infrastructures; The Sigrid Juselius Foundation (Finland); Torsten and Ragnar Söderberg Foundation (Sweden) (MT33/09); University Hospital Essen (Germany); University of Troms $\varnothing$ (Norway); Uppsala University (Sweden); Uppsala University Hospital (Sweden); Wellcome Trust (GR072960; 076113, 077016, 081682, 083948, 083270, 084711, 086596, 090367, 090532, 098051). A more detailed set of acknowledgements is provided in the Supplementary Note. 


\section{REFERENCES}

1. Stumvoll M, et al. Type 2 diabetes: principles of pathogenesis and therapy. Lancet. 2005; 365:13331346. [PubMed: 15823385]

2. Zeggini E, et al. Meta-analysis of genome-wide association data and large-scale replication identified additional susceptibility loci for type 2 diabetes. Nat. Genet. 2008; 40:638-645. [PubMed: 18372903]

3. Kong A, et al. Parental origin of sequence variants associated with complex diseases. Nature. 2009; 462:868-874. [PubMed: 20016592]

4. Voight BF, et al. Twelve type 2 diabetes susceptibility loci identified through large scale association analysis. Nat. Genet. 2010; 42:579-589. [PubMed: 20581827]

5. Dupuis J, et al. New genetic loci implicated in fasting glucose homeostasis and their impact on type 2 diabetes risk. Nat. Genet. 2010; 42:105-116. [PubMed: 20081858]

6. Qi L, et al. Genetic variants at 2 q24 are associated with susceptibility to type 2 diabetes. Hum. Mol. Genet. 2010; 19:2706-2715. [PubMed: 20418489]

7. Tsai F-J, et al. A genome-wide association study identifies susceptibility variants for type 2 diabetes in Han Chinese. PLoS Genet. 2010; 6:e1000847. [PubMed: 20174558]

8. Shu XO, et al. Identification of new genetic risk variants for type 2 diabetes. PLoS Genet. 2010; 6:e1001127. [PubMed: 20862305]

9. Yamauchi T, et al. A genome-wide association study in the Japanese population identifies susceptibility loci for type 2 diabetes at $U B E 2 E 2$ and $C 2 C D 4 A-C 2 C D 4 B$. Nat. Genet. 2010; 42:864-868. [PubMed: 20818381]

10. Kooner JS, et al. Genome-wide association study in individuals of South Asian ancestry identifies six new type 2 diabetes susceptibility loci. Nat. Genet. 2011; 43:984-989. [PubMed: 21874001]

11. Cho YS, et al. Meta-analysis of genome-wide association studies identifies eight new loci for type 2 diabetes in East Asians. Nat. Genet. 2012; 44:67-72. [PubMed: 22158537]

12. Voight BF, et al. The Metabochip, a custom genotyping array for genetic studies of metabolic, cardiovascular, and anthropometric traits. PLoS Genet. in press.

13. The 1000 Genomes Project Consortium. A map of human genome variation from population-scale sequencing. Nature. 2010; 467:1061-1073. [PubMed: 20981092]

14. Loos RJF, et al. Common variants near $M C 4 R$ are associated with fat mass, weight and risk of obesity. Nat. Genet. 2008; 40:768-775. [PubMed: 18454148]

15. Speliotes EK, et al. Association analyses of 249,796 individuals reveal 18 new loci associated with body mass index. Nat. Genet. 2010; 42:937-948. [PubMed: 20935630]

16. Teslovich TM, et al. Biological, clinical and population relevance of 95 loci for blood lipids. Nature. 2010; 466:707-713. [PubMed: 20686565]

17. Chambers JC, et al. Common genetic variation near $M C 4 R$ is associated with waist circumference and insulin resistance. Nat. Genet. 2008; 40:716-718. [PubMed: 18454146]

18. Heid IM, et al. Meta-analysis identifies 12 new loci associated with waist-hip ratio and reveals sexual dimorphism in the genetic basis of fat distribution. Nat. Genet. 2010; 42:949-960. [PubMed: 20935629]

19. Soranzo N, et al. Common variants at 10 genomic loci influence Hemoglobin A1C levels via glycaemic and nonglycaemic pathways. Diabetes. 2010; 59:3229-3239. [PubMed: 20858683]

20. Wray NR, et al. The genetic interpretation of area under the ROC curve in genomic profiling. PLoS Genet. 2010; 6:e1000864. [PubMed: 20195508]

21. Yang J, et al. Common SNPs explain a large proportion of the heritability for human height. Nat. Genet. 2010; 42:565-569. [PubMed: 20562875]

22. Lee SH, et al. Estimating missing heritability for disease from genome-wide association studies. Am. J. Hum. Genet. 2011; 88:294-305. [PubMed: 21376301]

23. Stahl EA, et al. Bayesian inference analyses of the polygenic architecture of rheumatoid arthritis. Nat. Genet. 2012; 44:483-489. [PubMed: 22446960]

24. Unoki H, et al. SNPs in $K C N Q 1$ are associated with susceptibility to type 2 diabetes in East Asian and European populations. Nat. Genet. 2008; 40:1098-1102. [PubMed: 18711366] 
25. Zeggini E, et al. Replication of genome-wide association signals in UK samples reveals risk loci for type 2 diabetes. Science. 2007; 316:1336-1341. [PubMed: 17463249]

26. Shea J, et al. Comparing strategies to fine-map the association of common SNPs at chromosome 9p21 with type 2 diabetes and myocardial infarction. Nat. Genet. 2011; 43:801-805. [PubMed: 21775993]

27. Yang $\mathbf{J}$, et al. Conditional and joint multiple-SNP analysis of GWAS summary statistics identifies additional variants influencing complex traits. Nat. Genet. 2012; 44:369-375. [PubMed: 22426310]

28. Dickson SP, et al. Rare variants create synthetic genome-wide associations. PLoS Biol. 2010; 8:e1000294. [PubMed: 20126254]

29. The International HapMap Consortium. A second generation human haplotype map of over 3.1 million SNPs. Nature. 2007; 449:851-861. [PubMed: 17943122]

30. The International HapMap Consortium. Integrating common and rare genetic variation in diverse human populations. Nature. 2010; 467:52-58. [PubMed: 20811451]

31. Waters KM, et al. Consistent association of type 2 diabetes risk variants found in Europeans in diverse racial and ethnic groups. PLoS Genet. 2010; 6:e1001078. [PubMed: 20865176]

32. Magi R, et al. Meta-analysis of sex-specific genome-wide association studies. Genet. Epidemiol. 2010; 34:846-853. [PubMed: 21104887]

33. Saxena $\mathrm{R}$, et al. Genetic variation in GIPR influences the glucose and insulin responses to an oral glucose challenge. Nat. Genet. 2010; 42:142-148. [PubMed: 20081857]

34. Scott RA, et al. Large-scale association analyses identify new loci influencing glycemic traits and provide insight into the underlying biological pathways. Nat. Genet. (in press).

35. Barrett JC, et al. Genome-wide association study and meta-analysis find that over 40 loci affect risk of type 1 diabetes. Nat. Genet. 2009; 41:703-707. [PubMed: 19430480]

36. Emilsson V, et al. Genetics of gene expression and its effect on disease. Nature. 2008; 452:423428. [PubMed: 18344981]

37. Ng PC, Henikoff S. SIFT: predicting amino acid changes that affect protein function. Nucleic Acids Res. 2003; 31:3812-4. [PubMed: 12824425]

38. Doria A, et al. The emerging genetic architecture of type 2 diabetes. Cell Metab. 2008; 8:186-200. [PubMed: 18762020]

39. Lage K, et al. A human phenome-interactome network of protein complexes implicated in genetic disorders. Nat. Biotechnol. 2007; 25:309-316. [PubMed: 17344885]

40. Lage K, et al. A large-scale analysis of tissue-specific pathology and gene expression of human disease genes and complexes. Proc. Natl. Acad. Sci. USA. 2008; 105:20870-20875. [PubMed: 19104045]

41. Rossin EJ, et al. Proteins encoded in genomic regions associated with immune-mediated disease physically interact and suggest underlying biology. PLoS Genet. 2011; 13:e1001273. [PubMed: 21249183]

42. Raychaudhuri $\mathrm{S}$, et al. Identifying relationships among genomic disease regions: predicting genes at pathogenic SNP associations and rare deletions. PLoS Genet. 2009; 5:e1000534. [PubMed: 19557189]

43. Muoio DM, Newgard CB. Mechanisms of disease: molecular and metabolic mechanisms of insulin resistance and beta-cell failure in type 2 diabetes. Nat. Rev. Mol. Cell. Biol. 2008; 9:193-205. [PubMed: 18200017]

44. Gangwisch JE. Epidemiological evidence for the links between sleep, circadian rhythms and metabolism. Obesity Rev. 2009; 10:37-45.

45. Boucher BJ. Vitamin D insufficiency and diabetes risks. Current Drug Targets. 2011; 12:61-87. [PubMed: 20795936]

46. Segre AV, et al. Common inherited variation in mitochondrial genes is not enriched for associations with type 2 diabetes or related glycemic traits. PLoS Genet. 2010; 12:e1001058. [PubMed: 20714348]

47. Pittas AG, et al. Adipocytokines and insulin resistance. J. Clin. Endocrinol. Metab. 2004; 89:447452. [PubMed: 14764746] 
48. Rane SG, et al. Loss of Cdk4 expression causes insulin-deficient diabetes and Cdk4 activation results in beta-islet cell hyperplasia. Nat. Genet. 1999; 22:44-52. [PubMed: 10319860]

49. Fiaschi-Taesch NM, et al. Induction of beta-cell proliferation and engraftment using a single G1/S regulatory molecule, cdk6. Diabetes. 2010; 59:1926-1936. [PubMed: 20668294]

50. Devlin B, Roeder K. Genomic control for association studies. Biometrics. 1999; 55:997-1004. [PubMed: 11315092]

51. Ioannidis J, et al. Heterogeneity in meta-analyses of genome-wide association investigations. PLoS One. 2007; 2

52. Lin S, et al. Exhaustive allelic transmission disequilibrium tests as a new approach to genome-wide association studies. Nat. Genet. 2004; 36:1181-1188. [PubMed: 15502828]

53. Storey JD, Tibshirani R. Statistical methods for identifying differentially expressed genes in DNA microarrays. Methods Mol. Biol. 2003; 224:149-157. [PubMed: 12710672]

54. Stolerman ES, et al. TCF7L2 variants are associated with increased proinsulin/insulin ratios but not obesity traits in the Framingham Heart Study. Diabetologia. 2009; 52:614-620. [PubMed: 19183934]

55. Stefansson H, et al. A common inversion under selection in Europeans. Nat. Genet. 2005; 37:129137. [PubMed: 15654335] 


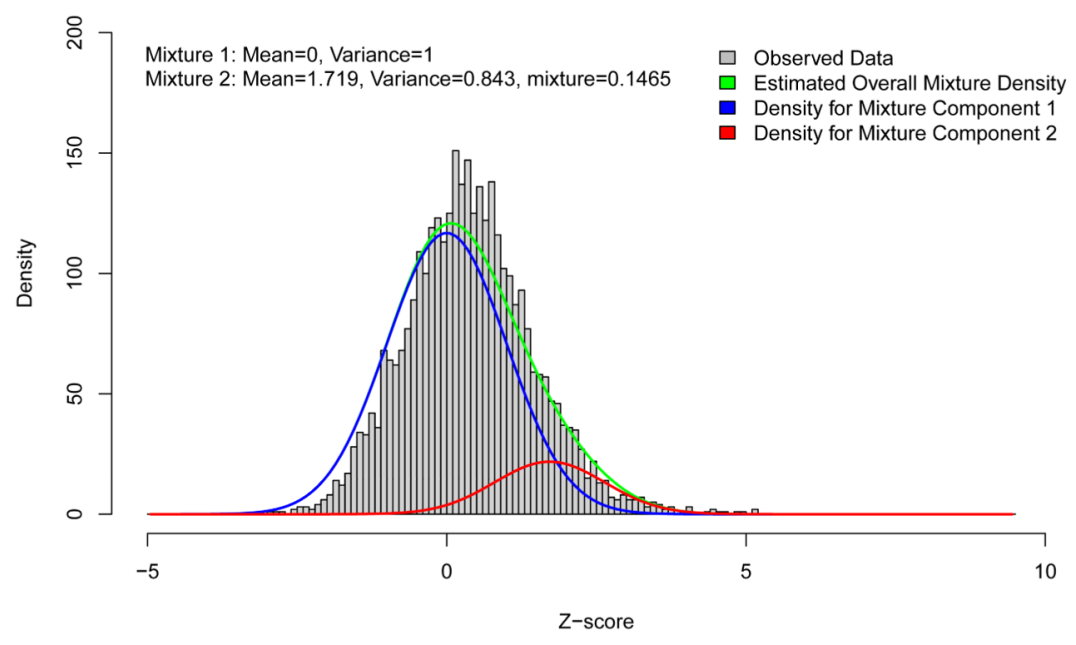

Figure 1.

Distribution of $Z$-scores from the Stage 2 meta-analysis, aligned to the risk allele from Stage 1. $Z$-scores were calculated at a subset of 3,412 independent T2D replication SNPs (CEU $r^{2}$ $<0.05$ ), excluding the 63 established and newly discovered autosomal susceptibility loci represented on Metabochip. The $Z$-score distribution is a mixture of: (i) the "null distribution" of SNPs having no effect on T2D (blue curve); and (ii) the "alternative distribution" of SNPs associated with the disease (red curve). 

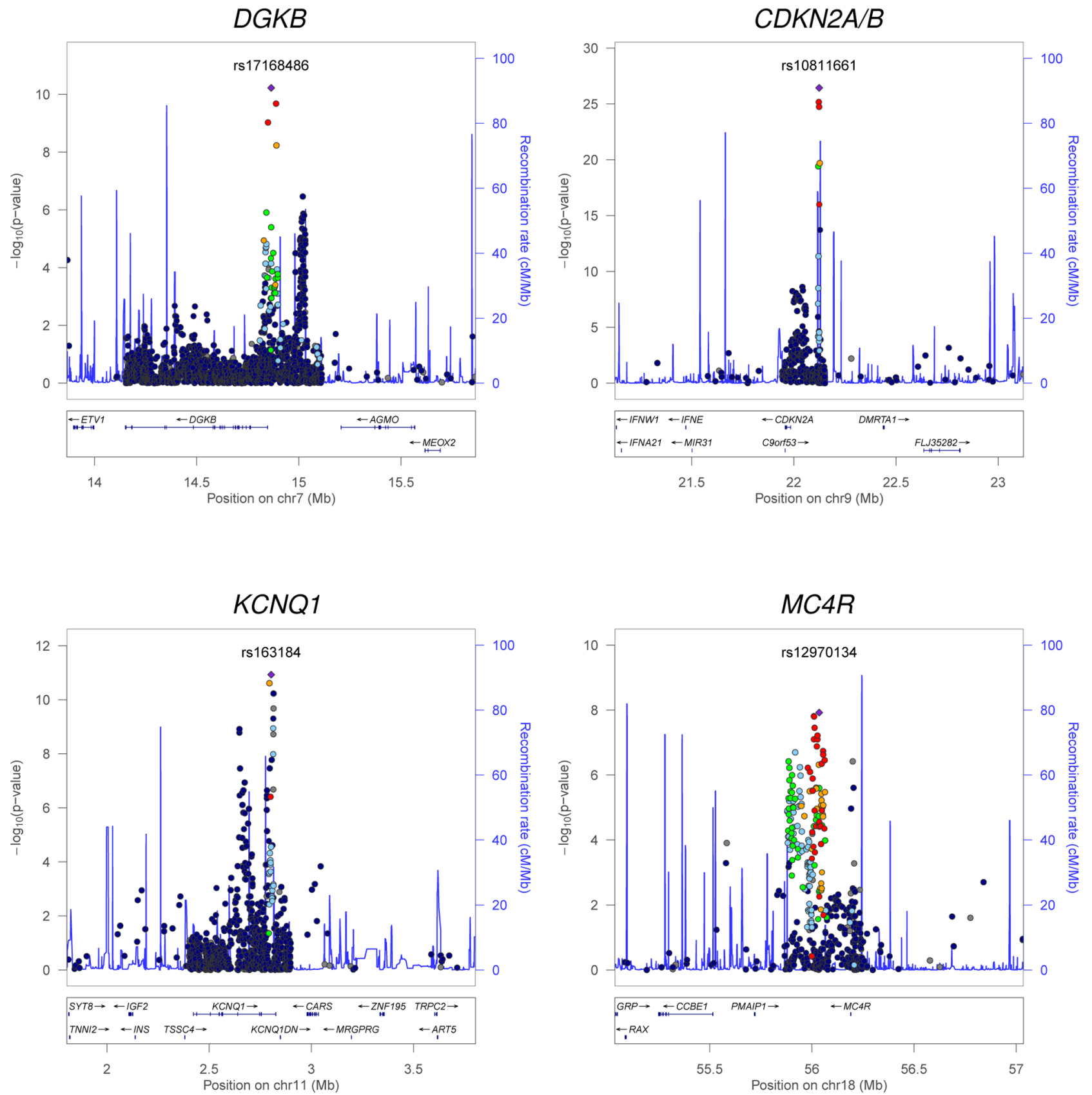

Figure 2.

Regional plots of T2D susceptibility loci with evidence of multiple association signals. Each point represents a Metabochip SNP passing quality control in our combined meta-analysis, plotted with their $P$-value (on a $-\log _{10}$ scale) as a function of genomic position (NCBI Build 36). In each panel, the lead SNP is represented by the purple diamond. The color coding of all other SNPs (circles) indicates LD with the lead SNP (estimated by CEU $r^{2}$ from the 1000 Genomes Project June 2010 release): red $r^{2} \geq 0.8$; gold $0.6 \leq r^{2}<0.8$; green $0.4 \leq r^{2}<0.6$; cyan $0.2 \leq r^{2}<0.4$; blue $r^{2}<0.2$; grey $r^{2}$ unknown. Recombination rates are estimated from the International HapMap Project and gene annotations are taken from the University of California Santa Cruz genome browser. 


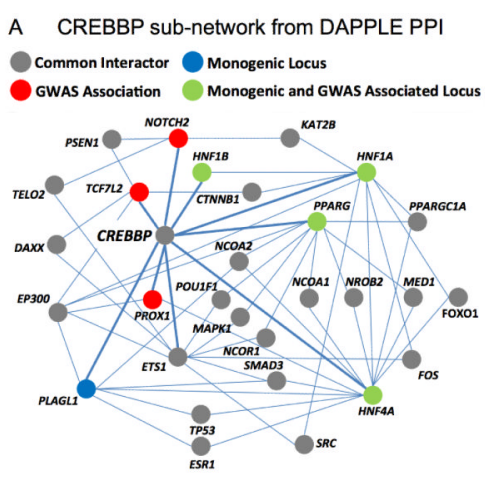

B GRAIL Connectivity Circle Plot

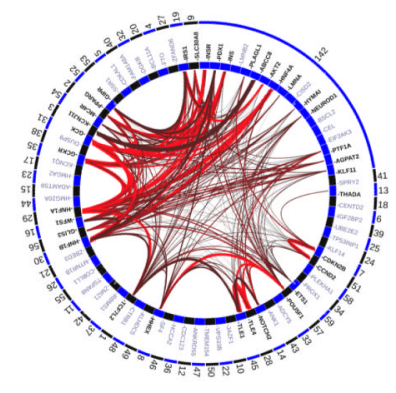

C Adipocytokine sub-network from DAPPLE PPI

common Interactor Monogenic Locus

GWAS Association Monogenic and GWAS Associated Locus

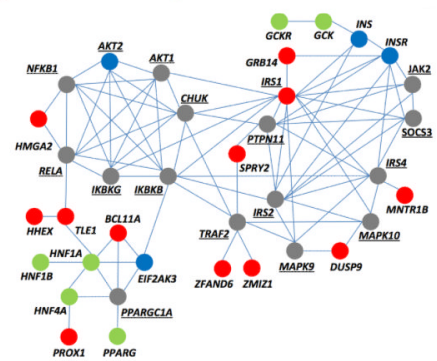

D GSEA of Adipocytokine signaling pathway

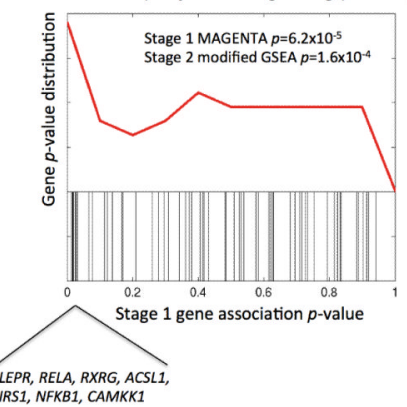

Figure 3.

Functional analyses. (a,b) Protein-protein interaction (PPI) sub-network for CREBBP and adipocytokine interactions. All direct interactions and common interactors between direct connections were extracted from the larger network of 314 proteins defined in the DAPPLE network analysis. Genes in the network are circles (nodes), colored according to the statistical relationship with T2D: common interactors between GWAS identified or monogenic loci are depicted as grey, monogenic loci (only) in blue, GWAS identified loci (only) in red, and loci with GWAS association and implicated by monogenic forms of diabetes are shown in green. Each interaction defined in the inWEB network is depicted by a line (edge) between nodes. (c) GRAIL circle plot of locus connectivity. Each locus is plotted in a circle where significant connections $(P<0.05)$ based on PubMed abstracts are drawn spanning the circle. Conservatively, we treated all monogenic loci (region 142) as a single locus by which connectivity is assessed. The strongest connections $(P<0.001)$ are colored in bright red. (d) GSEA of associations in the adipocytokine signaling pathway. The black bars represent the Stage 1 meta-analysis $P$-values of 63 autosomal genes in the Adipocytokine Signaling pathways (KEGG). A density plot of the black bars is depicted in the top panel (red line). The replicating genes in the leading edge of the GSEA are listed. The Stage 2 modified GSEA $P=1.6 \times 10^{-4}$ was calculated based on both the primary and secondary transcripts using the LD locus definition. 

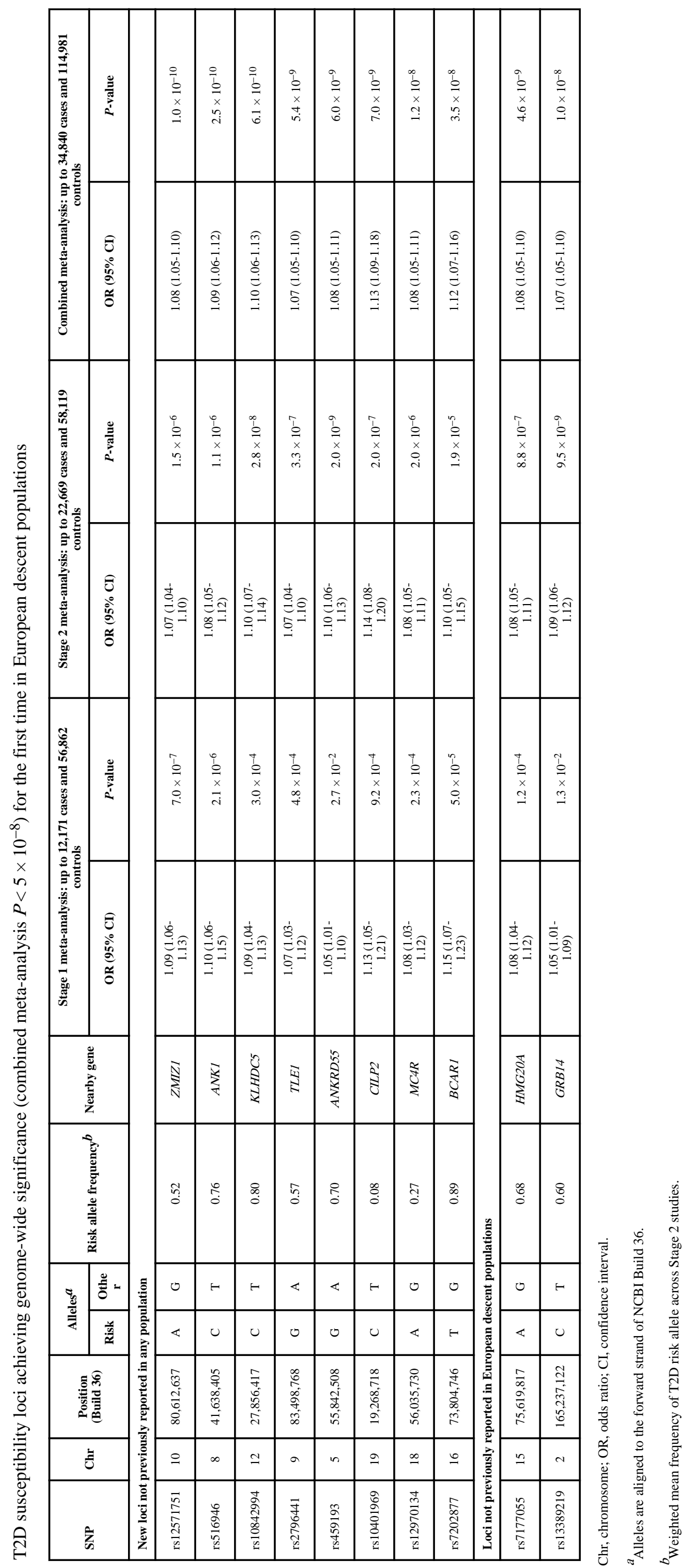

Nat Genet. Author manuscript; available in PMC 2013 February 12 


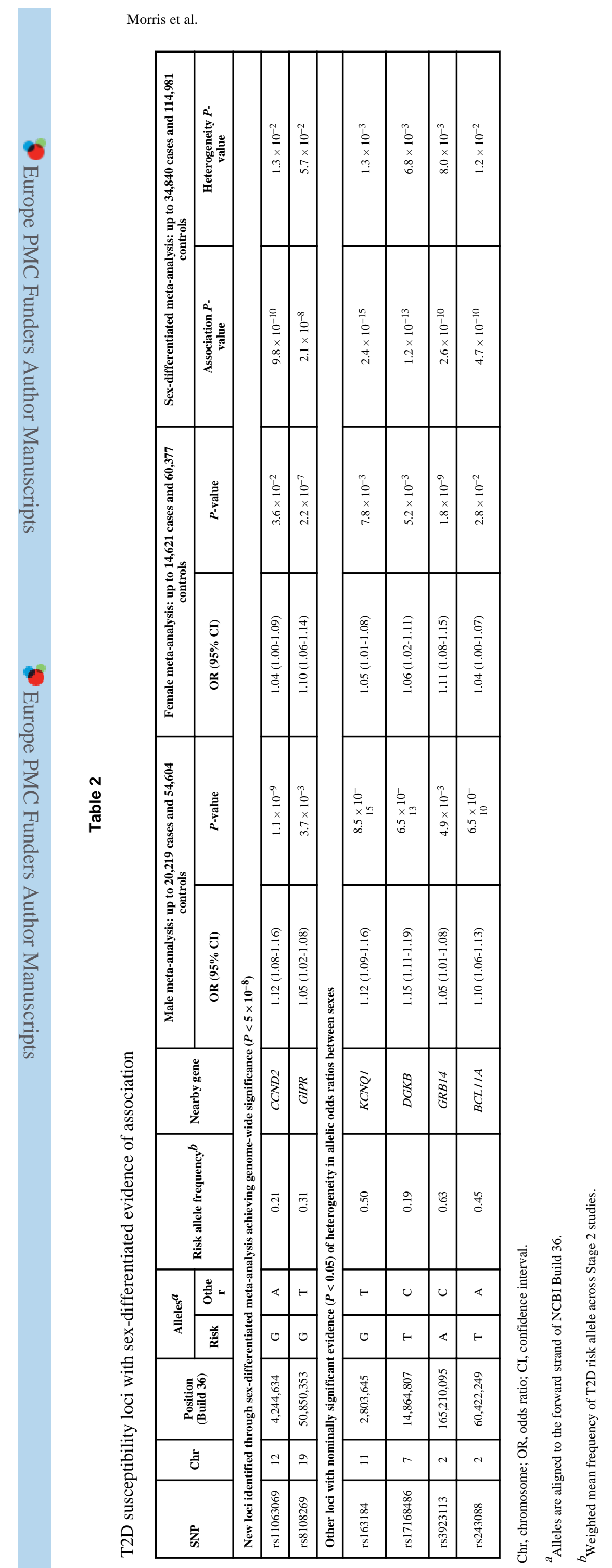

Page 26 